\title{
Mamíferos não voadores (Mammalia) da Estação Ecológica Serra Geral do Tocantins
}

\author{
Ana Paula Carmignotto ${ }^{1,3}$ \& Caroline Cotrim Aires $^{2}$ \\ ${ }^{1}$ Universidade Federal de São Carlos - UFSCar, Campus Sorocaba, Rod. João Leme dos Santos, \\ SP-264, Km 110, Bairro Itinga, CEP 18052-780, Sorocaba, SP, Brasil \\ ${ }^{2}$ Museu de Zoologia, Universidade de São Paulo - USP, Av. Nazaré, 481, Ipiranga, \\ CEP 04263-000, São Paulo, SP, Brasil \\ ${ }^{3}$ Autor para correspondência: Ana Paula Carmignotto,e-mail: apcarmig@ufscar.br
}

CARMIGNOTTO, A.P. \& AIRES, C.C. Non-volant mammals (Mammalia) from Estação Ecológica Serra Geral do Tocantins. Biota Neotrop. 11(1): http://www.biotaneotropica.org.br/v11n1/en/abstract?article+bn03911012011.

\begin{abstract}
The terrestrial mammal community was surveyed along three distinct localities (1-Mateiros, TO; 2- Rio da Conceição, TO e 3- Formosa do Rio Preto, BA) in the Estação Ecológica Serra Geral do Tocantins. The small non-volant mammals were recorded by the use of conventional live traps (5,396 trap.nights) and pitfall traps (5,300 trap.nights) in the different physiognomies found, such as: "campo úmido", "campo limpo", "campo sujo", "campo cerrado", "cerrado sensu stricto", "cerrado sensu stricto with rocks", gallery forest and wet gallery forest. The large mammals were casually registered by visual or indirect evidences (tracks, feces, carcasses). We recorded 24 small non-volant mammals and 17 large mammals, totalizing 41 species for this region. The small mammal community was characterized by several rare and intermediate in abundance species, and few species very abundant. Rodents from the family Cricetidae dominated both in number of species (14) as in number of individuals (50\% of the community). The species segregated among the forested and open formations, showing the great habitat selectivity and the importance to survey the habitat mosaic to characterize the diversity of this mammal group. The small non-volant mammal fauna surveyed showed some overlap with the fauna from the Caatinga and Amazon biomes, demonstrating the importance of these biomes in the faunal composition of the communities localized in the northern portion of the Cerrado. In addition, we also recorded endemic species and species with a restricted distribution to this north portion, characterizing a distinct community within the Cerrado. The large mammal community was characterized by a high number of threatened species (10), increasing the importance of this region in preserving the fauna of the northern portion of the Cerrado.
\end{abstract}

Keywords: marsupials, rodents, large mammals, diversity, composition, Cerrado, conservation.

CARMIGNOTTO, A.P. \& AIRES, C.C. Mamíferos não voadores (Mammalia) da Estação Ecológica Serra Geral do Tocantins. Biota Neotrop. 11(1): http://www.biotaneotropica.org.br/v11n1/pt/abstract?article+bn03911012011.

Resumo: A comunidade de mamíferos terrestres foi amostrada em três localidades (1-Mateiros, TO; 2- Rio da Conceição, TO e 3- Formosa do Rio Preto, BA) no interior da Estação Ecológica Serra Geral do Tocantins. Para o registro dos marsupiais e pequenos roedores foram utilizadas armadilhas convencionais (5.396 armadilhas. noite) e armadilhas de queda (5.300 pitfalls.noite) nas diferentes fitofisionomias encontradas, entre elas: campo úmido, campo limpo, campo sujo, campo cerrado, cerrado sensu stricto, cerrado com afloramentos rochosos, mata de galeria e mata de galeria úmida. No caso dos mamíferos de médio e grande porte, foram obtidos registros casuais através de observação direta e evidências indiretas (rastros, fezes, crânios e carcaças de animais encontrados mortos). Foram amostradas 24 espécies de pequenos mamíferos e 17 espécies de mamíferos de médio e grande porte, totalizando 41 espécies para a região. Considerando-se os pequenos mamíferos, a comunidade foi representada por várias espécies raras e de abundância intermediária, e poucas espécies muito abundantes. Os roedores cricetídeos dominaram tanto em número de espécies (14) quanto em abundância (50\% da comunidade). As espécies se distribuíram, basicamente, em dois tipos de fisionomias: um grupo esteve restrito aos ambientes florestais, e outro às formações abertas, demonstrando a grande seletividade de hábitats e a importância de se amostrar o mosaico de hábitats presente na região para uma melhor caracterização da diversidade deste grupo de mamíferos. Em termos biogeográficos, a fauna de pequenos mamíferos amostrada apresentou certa sobreposição com a fauna da Caatinga e da Amazônia, evidenciando a importância destes domínios para a composição de espécies das comunidades que habitam a porção norte do Cerrado, além da presença de espécies endêmicas e de distribuição geográfica restrita ao norte do domínio, caracterizando uma comunidade distinta de outras regiões do Cerrado. Em relação aos mamíferos de médio e grande porte, a presença de um elevado número de espécies ameaçadas de extinção (10) também ressalta a importância da preservação desta região.

Palavras-chave: marsupiais, roedores, mamíferos de médio e grande porte, diversidade, composição, Cerrado, conservação. 


\section{Introdução}

O Cerrado é o domínio aberto mais representativo da América do Sul, tanto em termos de área, abrangendo aproximadamente 2 milhões de $\mathrm{km}^{2}$, quanto em termos de diversidade, apresentando em torno de 10.000 espécies de plantas, 150 de anfíbios, 252 de répteis, 837 de aves e 249 espécies de mamíferos (Myers et al. 2000, Colli et al. 2002, Marinho-Filho et al. 2002, Carmignotto 2005, Costa et al. 2007, Zortéa \& Aguiar 2008). Entretanto, do ponto de vista científico, o Cerrado permanece como um dos domínios brasileiros menos estudados (Oliveira \& Marquis 2002). Em relação à mastofauna, à medida que inventários e trabalhos taxonômicos são realizados, novos gêneros e novas espécies são descritos para a região (e.g. Hershkovitz 1990a,b, 1993, Langguth \& Bonvicino 2002, Bonvicino 2003, Gonçalves et al. 2005, Weksler \& Bonvicino 2005), revelando a necessidade de estudos neste domínio.

À parte os problemas científicos, o Cerrado vem enfrentando um período de intensa fragmentação e degradação. Durante os últimos 40 anos, a região tem sido utilizada para criação extensiva de gado e para o desenvolvimento da agricultura moderna, recebendo vários incentivos econômicos como parte de políticas federais que têm como objetivo povoar e transformá-la em um dos principais pólos agrícolas do país (Marris 2005). Estima-se que $80 \%$ da vegetação nativa foi transformada em pastos cultiváveis, áreas de agricultura, usinas hidrelétricas, assentamentos urbanos ou simplesmente áreas degradadas (Klink \& Machado 2005). No entanto, apesar da urgência em se preservar áreas de Cerrado, atualmente apenas 2,2\% de sua área total está protegida por lei, em unidades de conservação federais (Cavalcanti \& Joly 2002). Para embasar a escolha de áreas prioritárias para conservação, é necessário que a fauna do Cerrado seja conhecida, bem como os limites da distribuição geográfica das espécies que aí ocorrem.

A região da Estação Ecológica Serra Geral do Tocantins (EESGT) está parcialmente inserida no polígono de importância extremamente alta para a conservação da biodiversidade, denominado "Águas Emendadas do Rio do Sono", reconhecido pelo workshop "Ações Prioritárias para a Conservação do Cerrado e Pantanal" de março de 1998. A Estação também é uma das áreas nucleares da Reserva da Biosfera do Cerrado, e compõe parte do Corredor Ecológico do Jalapão (IBAMA 2004). O estudo da fauna de mamíferos desta região justifica-se por tratar-se de uma área na porção norte do Cerrado, com influência de outros domínios como a Caatinga e a Floresta Amazônica, com potencial de endemismos (Carmignotto 2005). Esta área, muito bem preservada, não foi satisfatoriamente amostrada até o momento.

Os objetivos deste estudo foram inventariar a fauna de mamíferos terrestres da região, acrescentando informações sobre abundância e seletividade de habitat das espécies nos sítios de coleta, no sentido de contribuir com o aumento do conhecimento sobre a fauna de mamíferos do Cerrado e de tornar possíveis comparações com domínios adjacentes.

\section{Material e Métodos}

\section{1. Área de estudo}

A EESGT localiza-se na porção norte do Cerrado, a leste do Estado de Tocantins, e extremo noroeste da Bahia (entre $10^{\circ} 30^{\prime}$ a $11^{\circ} 17^{\prime} \mathrm{S}$ e $47^{\circ} 14^{\prime}$ a $46^{\circ} 10^{\prime} \mathrm{W}$ - $496 \mathrm{~m}$ ). Com uma área de aproximadamente 716.306 ha, situa-se a $260 \mathrm{~km}$ a leste de Palmas, Tocantins, abrangendo três municípios nesse estado (Ponte Alta do Tocantins a noroeste, Mateiros a nordeste, e Rio da Conceição ao sul) e um município na Bahia (Formosa do Rio Preto) (Figura 1).
A EESGT situa-se em uma região caracterizada pelo relevo levemente ondulado a ondulado, com predomínio das coberturas metassedimentares conhecidas como Patamares dos rios São Francisco-Tocantins. A paisagem é composta por planaltos e chapadões aplainados, serras do tipo "mesa" e morros testemunhos (Mamede et al. 2002, Villela \& Nogueira neste volume). A região apresenta relativa estabilidade geológica, porém sofre ação erosiva intensa que constantemente modifica a paisagem regional. A formação de dunas, por exemplo, devido à ação fluvial e eólica sobre os chapadões areníticos é característica. Os solos predominantes são os areno-quartzosos distróficos e álicos, mas também se registra a presença de latossolos na região (Mamede et al. 2002, IBAMA 2004).

A EESGT localiza-se próxima às nascentes do rio Novo, da sub-bacia do rio do Sono, que por sua vez é a origem dos principais tributários da bacia hidrográfica do Araguaia-Tocantins (Mamede et al. 2002). O clima dominante é o Tropical semi-úmido do Brasil Central (Aw segundo Köppen). A região amostrada apresenta precipitação média anual em torno de $1450 \mathrm{~mm}$, com maior pluviosidade $(263 \mathrm{~mm})$ no mês de dezembro, e menor $(0 \mathrm{~mm})$ nos meses de junho e julho. Na estação chuvosa, entre outubro a abril, registra-se excedente hídrico em torno de $700 \mathrm{~mm}$; na estação seca, entre maio a setembro, há uma deficiência hídrica de $185 \mathrm{~mm}$. A temperatura média anual é de $24,1{ }^{\circ} \mathrm{C}$; registrando-se as mais altas temperaturas ao final da estação seca e início da estação chuvosa, nos meses de setembro e outubro (média de $25,6{ }^{\circ} \mathrm{C}$ ), e as mais baixas ao longo da estação seca, em julho (média de 22,6 ${ }^{\circ} \mathrm{C}$ ) (Leemans \& Cramer 1991) (Figura 2).

Segundo o mapa de vegetação do IBGE (IBGE 1993), o tipo de fisionomia dominante é a Savana (Cerrado), e na região ocorrem os tipos de hábitat conhecidos como savana gramíneo-lenhosa, savana parque e savana arborizada, que correspondem às fisionomias de campo limpo, campo sujo, campo cerrado e cerrado sensu stricto (Oliveira \& Ratter 2002). Os tipos de vegetação dominantes são o campo limpo e campo sujo entremeados por manchas de campo cerrado e cerrado sensu stricto. É também muito comum observarmos a presença de extensas veredas e campos úmidos no interior das áreas de campo. Formações florestais, no entanto, são escassas, encontrando-se áreas de mata de encosta e mata de galeria próximas aos maiores rios, e em alguns trechos em suas margens, como no Rio Novo.

A região encontra-se em ótimo estado de conservação, apresentando grandes extensões inabitadas e uma das mais baixas densidades demográficas do país, com apenas 1,3 habitantes $/ \mathrm{km}^{2}$ (IBAMA 2004). Há pequenos povoados e fazendas de criação de gado ou de cultivo nas proximidades e em seu interior. Entretanto, a caça e as atividades turísticas parecem ser as atividades mais relacionadas à perturbação ambiental na região.

\section{Metodologia de amostragem}

A comunidade de mamíferos terrestres foi amostrada em três localidades no interior da EESGT. Na primeira excursão, de 29 de março a 9 de abril de 2003, foram amostradas áreas situadas em torno de $60 \mathrm{~km}$ da cidade de Mateiros, TO (localidade 1 - $10^{\circ} 40^{\prime} \mathrm{S}$ e $46^{\circ} 52^{\prime}$ W). Na segunda viagem de campo, de 26 de janeiro a 4 de fevereiro de 2008, foram amostradas áreas localizadas em torno de $70 \mathrm{~km}$ da cidade de Rio da Conceição, TO (localidade $2-11^{\circ} 14^{\prime}$ S e $46^{\circ} 51^{\prime}$ W). A terceira excursão, de 9 a 16 de fevereiro de 2008 , concentrou-se em áreas próximas ao município de Formosa do Rio Preto, BA (localidade $3-10^{\circ} 38^{\prime} \mathrm{S}$ e $46^{\circ} 10^{\prime} \mathrm{W}$ ) (Figura 1). As amostragens ocorreram durante a estação chuvosa com o intuito de obter dados comparativos visto que a primeira localidade foi amostrada durante a estação chuvosa. Em outras amostragens realizadas no Cerrado, observa-se um maior sucesso de captura e 


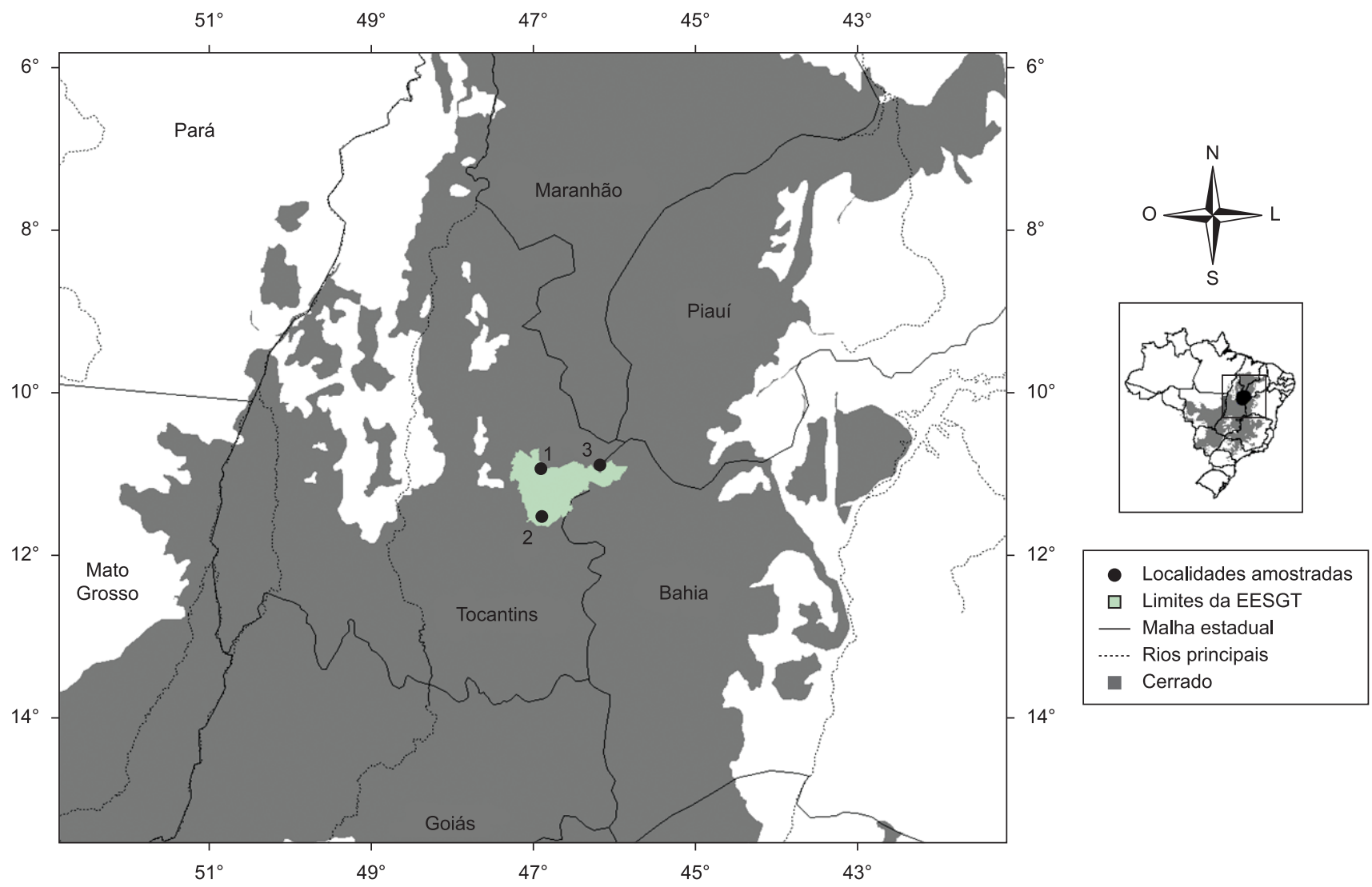

Figura 1. Mapa mostrando a localização dos pontos amostrados no Brasil, e no detalhe no interior da EESGT. $1=$ Mateiros, TO; $2=$ Rio da Conceição, TO e 3 = Formosa do Rio Preto, BA. A distribuição do Cerrado baseia-se em IBGE (IBGE 1993).

Figure 1. Map showing the points surveyed in Brazil and in detail in the EESGT. 1 = Mateiros, TO; 2 = Rio da Conceição, TO and $3=$ Formosa do Rio Preto, BA. The Cerrado distribution is based on IBGE (IBGE 1993).

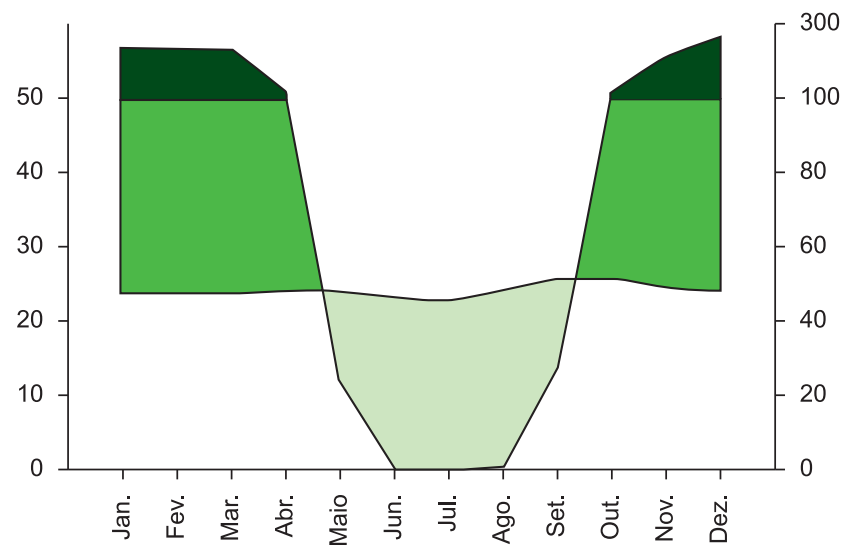

Figura 2. Diagrama climático segundo Walter (1986) da região onde se insere a EESGT.

Figure 2. Climatic diagram based on Walter (1986) from the region of EESGT.

riqueza de espécies durante esta época do ano (Marinho-Filho et al. 1994, Vieira \& Palma 2005). Cada localidade foi amostrada por períodos de 8 a 10 dias de captura, totalizando 28 dias de amostragem durante o período em campo.

Foram amostradas diferentes fitofisionomias encontradas nas localidades estudadas e cada ponto de amostragem foi georreferenciado e classificado quanto ao tipo de hábitat, considerando-se os tipos básicos de vegetação já reconhecidos para o Cerrado (ver Eiten 1994, Oliveira \& Ratter 2002).

\subsection{Pequenos mamíferos não voadores}

Dada a variação em tamanho registrada entre pequenos mamíferos (Eisenberg \& Redford 1999) e as diferenças de comportamento frente às armadilhas (Woodman et al. 1996), foram utilizados diferentes modelos de armadilhas complementares: 1- armadilhas de interceptação e queda ou pitfalls, e 2- armadilhas de isca, da marca Sherman $^{\mathrm{TM}}$ (de dois tamanhos: 7,5 $\times 8,5 \times 23 \mathrm{~cm}$ e $10 \times 12 \times 37,5 \mathrm{~cm}$ ); e gaiolas de fabricação artesanal, com $19,5 \times 20 \times 32 \mathrm{~cm}$. O uso de métodos complementares em um levantamento faunístico permite que a amostragem realizada seja mais eficiente, já que possibilita a captura de um maior número de espécies em um intervalo de tempo menor (Lyra-Jorge \& Pivello 2001, Umetsu et al. 2006). A configuração dos sistemas de disposição das armadilhas foi a seguinte:

Pitfalls: foram dispostos em linhas independentes de cinco (localidades 2 e 3 ) ou dez (localidade 1) estações de captura cada uma, sendo cada estação disposta a 15 metros de distância uma da outra. Cada estação foi composta por quatro baldes de $35 \mathrm{~L}$ enterrados no solo, sendo um central e os outros três dispostos a quatro metros de distância deste, nos vértices de um triângulo equilátero imaginário. Uma lona de $50 \mathrm{~cm}$ de altura foi esticada perpendicularmente ao solo, unindo o balde central aos outros três e funcionando como cercas-guia, como descrito na disposição radial em Cechin \& Martins (2000). Em cada localidade amostrada foram instaladas seis (localidade 1 ) ou nove (localidades 2 e 3 ) linhas de pitfalls, cada uma contendo 40 e 20 baldes respectivamente, totalizando 
240 (localidade 1) e 180 baldes (localidades 2 e 3). A configuração utilizada já foi instalada em outras localidades apresentando resultados satisfatórios e superando, inclusive, o sucesso de captura das armadilhas convencionais (Hice \& Schmidly 2002, Umetsu et al. 2006, Cáceres et al. 2011).

Armadilhas convencionais: foram instaladas em linhas independentes compostas de 15 a 30 armadilhas cada. As armadilhas de modelos diferentes ("Sherman pequena", "Sherman grande" e "gaiola") foram intercaladas, sendo a distância entre armadilhas consecutivas de $15 \mathrm{~m}$. Na localidade 1 foram utilizadas 210 armadilhas (7 linhas de 30 armadilhas), na localidade 2 foram usadas 195 armadilhas (6 linhas de 30 e 1 linha de 15 armadilhas) e na localidade 3 instalamos 189 armadilhas (9 linhas de 21 armadilhas). As armadilhas também foram dispostas a alturas diferentes, do nível do solo até $2 \mathrm{~m}$, com o intuito de amostrar espécies de hábito escansorial ou arborícola (e.g. Astúa et al. 2006). A isca utilizada visou atrair espécies que apresentam dietas variadas (Patric 1970, Astúa et al. 2006), e foi constituída por uma mistura de pasta de amendoim, sardinha e fubá, sendo que nas gaiolas uma rodela de mandioca foi colocada como suporte.

As unidades de esforço empregadas foram a) pitfalls.noite e b) armadilhas.noite, as quais foram obtidas multiplicando-se o número total de armadilhas e pitfalls (baldes) pelo número total de noites em que os mesmos estiveram abertos. O esforço total empreendido em cada metodologia de amostragem por localidade encontra-se descrito na Tabela 1. A eficiência das duas metodologias de captura empregadas foi expressa pelo sucesso de captura, em porcentagem (Wilson et al. 1996), dividindo-se o número total de indivíduos capturados pelo esforço total empregado durante o período de estudo.

\subsection{Mamíferos de médio e grande porte}

Registros obtidos através de observação direta e evidências indiretas (rastros, fezes, crânios e carcaças de animais encontrados mortos) foram considerados, apesar de seu esforço não poder ser quantificado, sendo estes dados obtidos ocasionalmente durante o período em campo (Voss \& Emmons 1996). Os rastros foram fotografados, mensurados e identificados geralmente ao nível genérico, e ao nível específico quando possível, utilizando-se como referência Becker \& Dalponte (1991) e Borges \& Tomás (2004).

\section{Preparação e destino dos exemplares}

Os espécimes de mamíferos coletados foram preservados em via líquida (formol 10\%), e em via seca (taxidermizados), sendo a carcaça nesse caso preservada em álcool $70 \%$ e posteriormente preparada como crânio e esqueleto pós-craniano em dermestário. Cada espécime foi individualizado por etiqueta numerada e a data, o local de coleta, a espécie, o sexo, a classe etária, a condição reprodutiva, e as medidas padrão (vide Moojen 1943, Emmons \& Feer 1997) foram registradas. Alíquotas de tecido visceral dos espécimes coletados encontram-se preservadas em etanol absoluto para futuros estudos moleculares. O material coletado (espécimes e tecidos) está depositado na coleção de mamíferos do Museu de Zoologia da Universidade de São Paulo (MZUSP), em processo de tombamento (ver Apêndice 1). Os exemplares excedentes, ou seja, capturados em número superior a 15 indivíduos por espécie e por localidade, e que tiveram sua identidade taxonômica confirmada em campo, foram marcados através de perfurações nas orelhas, e soltos no local de captura (Wilson et al. 1996). As mesmas informações já citadas para os espécimes coletados foram obtidas para os exemplares marcados e soltos.

A identificação taxonômica das espécies foi realizada através da análise da pele e do crânio dos espécimes coletados, sendo utilizada como referência a coleção de mamíferos do MZUSP, bem como a literatura científica especializada. A nomenclatura das espécies e o arranjo taxonômico nas categorias de ordem, família e subfamília seguem Wilson \& Reeder (2005), exceto quando artigos mais recentes alteraram a nomenclatura e o arranjo publicados nessa obra, como, por exemplo, Voss et al. (2005), Weksler \& Bonvicino (2005) e Weksler et al. (2006). O arranjo taxonômico na categoria de tribos para os roedores sigmodontíneos segue Smith \& Patton (1999).

\section{Análise dos dados}

A suficiência do esforço de captura foi avaliada através das curvas de rarefação obtidas para cada localidade, assim como para o total da área amostrada, como uma maneira de avaliar a amostragem realizada no presente estudo (Gotelli \& Colwell 2001). Utilizamos o estimador Jackknife de segunda ordem para estimar a riqueza esperada, visto que apresenta bons resultados em comunidades com baixo índice de equabilidade, como encontrado no presente estudo (Brose et al. 2003). Utilizamos o programa ESTIMATE S versão 8.0.0 (Colwell 2004) para estimar os valores das curvas de rarefação, e do estimador de riqueza. Estes dados foram estimados utilizando 100 sorteios aleatórios sem reposição das sequiências de amostras. A média e o desvio padrão da riqueza observada e estimada foi apresentada para cada localidade e para a amostragem como um todo.

Utilizamos o conceito de soma (additive partitioning) (Loreau 2000, Veech et al. 2002) para estimar e comparar os diferentes níveis de diversidade, expressos em porcentagem, permitindo avaliar a contribuição relativa de cada um (diversidade alfa e beta) para a diversidade total (diversidade gama) (Whittaker 1972). A diversidade alfa foi calculada como o número médio de espécies amostrado em cada hábitat (ou a riqueza de cada localidade); a diversidade beta como o número médio de espécies encontrado entre hábitats (ou a riqueza entre localidades); e a diversidade gama como o número total de espécies obtido na área de estudo.

A abundância relativa das espécies foi estimada dividindo-se o número total de indivíduos amostrados de cada espécie pelo número total de indivíduos registrados em cada localidade, e na área de estudo como um todo (Magurran 2004).

O índice de diversidade de Shannon-Wiener (H') foi utilizado como medida de diversidade (Krebs 1999). Estimamos também a equabilidade da amostra $\left(\mathrm{E}=\mathrm{e}^{\mathrm{H}^{\prime}} / \mathrm{S}\right.$, onde $\mathrm{S}$ é o número total de espécies registradas) como medida da contribuição das espécies amostradas para a abundância total. Estes índices foram usados de maneira comparativa entre as localidades, e também foram estimados para a amostragem como um todo.

Devido ao esforço não ter sido semelhante entre hábitats amostrados, utilizou-se como medida de comparação o sucesso de captura expresso em porcentagem (\%), calculado dividindo-se o número total de indivíduos de cada espécie capturada em cada hábitat pelo esforço aplicado neste hábitat (número de armadilhas ou de pitfalls, ou de ambos, quando a espécie foi capturada pelos dois tipos de armadilhas).

A ordenação por Análise de Correspondência Retificada (Detrended Correspondence Analysis - DCA) foi utilizada com o intuito de verificar a relação entre os diferentes hábitats quanto à composição e abundância (baseada no sucesso de captura) das espécies de pequenos mamíferos. Este método foi escolhido visto que a ordenação dos hábitats e das espécies pode ser visualizada simultaneamente, facilitando a interpretação do resultado, além de ser o método de ordenação recomendado quando a relação entre as espécies não é linear, como no presente estudo (Urban 2000). Esta análise foi realizada pelo programa PC-ORD for Windows versão 4.25 (McCune \& Mefford 1999), onde utilizamos a opção eixos reescalonados e 26 como o número de segmentos, considerados satisfatórios neste tipo de análise (Palmer 2004). 
Tabela 1. Linhas de armadilhas convencionais e de pitfalls instaladas nas três localidades amostradas na EESGT indicando o esforço de captura (armadilhas. noite e pitfalls.noite) por hábitat em cada localidade amostrada e na área de estudo.

Table 1. Live and pitfall trap lines installed in the three localities surveyed in the EESGT indicating the sampling effort (trap.nights and pitfall.nights) in each habitat and locality in the study area.

\begin{tabular}{|c|c|c|c|c|c|c|}
\hline \multirow[t]{2}{*}{ Hábitats } & \multicolumn{2}{|c|}{ Localidade 1} & \multicolumn{2}{|c|}{ Localidade 2} & \multicolumn{2}{|c|}{ Localidade 3} \\
\hline & Arm. & Pitfalls & Arm. & Pitfalls & Arm. & Pitfalls \\
\hline Campo úmido / vereda & 305 & - & 270 & 180 & 189 & 180 \\
\hline Campo limpo & 275 & 760 & 240 & 160 & 378 & 360 \\
\hline Campo sujo & 275 & 360 & 270 & 180 & 378 & 360 \\
\hline Campo cerrado & 305 & - & 240 & 160 & 189 & 180 \\
\hline Cerrado sensu stricto & 305 & 400 & 270 & 360 & 189 & 180 \\
\hline Cerrado sensu stricto com afloramentos rochosos & 275 & 280 & 270 & 360 & 378 & 360 \\
\hline Mata galeria úmida & - & - & 120 & 160 & - & - \\
\hline Mata galeria & 275 & 320 & - & - & - & - \\
\hline Total & 2015 & 2120 & 1680 & 1560 & 1701 & 1620 \\
\hline Total & 5396 & 5300 & - & - & - & - \\
\hline
\end{tabular}

\section{Resultados}

\section{Pequenos mamíferos não voadores}

\subsection{Riqueza e composição de espécies}

A fauna de pequenos mamíferos não voadores amostrada na região da EESGT foi composta de 24 espécies: seis pertencentes à ordem Didelphimorphia, família Didelphidae, subfamília Didelphinae, e uma à subfamília Caluromyinae; 14 pertencentes à ordem Rodentia, família Cricetidae, subfamília Sigmodontinae; duas pertencentes à família Echimyidae, subfamília Eumysopinae; e 1 pertencente à família Caviidae, subfamília Caviinae (Tabela 2). A única espécie não identificada em nível específico (Thrichomys sp.) é uma espécie provavelmente nova. Bonvicino et al. (2002b) e Braggio \& Bonvicino (2004), baseado em dados citogenéticos $(2 \mathrm{n}=26$ e $\mathrm{NF}=48$ ) e moleculares (representando uma linhagem distinta), associaram espécimes provenientes do estado da Bahia ao táxon Thrichomys inermis. Os espécimes da EESGT apresentaram 2n $=26$ e NF $=46$, e segundo Basile (2003), não se enquadram na diagnose de T. inermis, táxon restrito à porção leste da Bahia, delimitado pelo rio São Francisco; sendo considerado um táxon distinto no presente estudo (táxon citado como Thrichomys sp. 2 em Carmignotto 2005).

$\mathrm{O}$ número de gêneros amostrados foi praticamente semelhante ao número de espécies, dado que somente os gêneros Cerradomys, Oecomys e Oligoryzomys apresentaram duas espécies cada um na região. Dentre os roedores cricetídeos, foram registradas espécies pertencentes às tribos Akodontini (Necromys lasiurus, Oxymycterus delator e Thalpomys cerradensis), Oryzomini (Cerradomys marinhus, C. scotti, Hylaeamys megacephalus, Nectomys rattus, Oecomys bicolor, O. paricola, Oligoryzomys fornesi e O. moojeni), Phyllotini (Calomys tener), Thomasomini (Rhipidomys macrurus) e um incertae sedis (Pseudoryzomys simplex).

\subsection{Comparação entre os métodos de amostragem}

As armadilhas de isca foram responsáveis pela captura de 135 indivíduos (20 marsupiais, 50 roedores cricetídeos, 64 equimídeos e 1 caviídeo), totalizando um sucesso de 2,5\% (Tabela 2). Os pitfalls apresentaram sucesso de captura inferior, de 1,3\% e amostraram 70 indivíduos (16 marsupiais, 53 roedores cricetídeos e 1 equimídeo) (Tabela 2). A composição de espécies amostradas pelas duas metodologias de captura também apresentou diferenças. As armadilhas de isca amostraram 16 espécies distintas, sendo dois marsupiais (Didelphis albiventris e Micoureus demerarae), dois roedores cricetídeos (Nectomys rattus e Rhipidomys macrurus), um equimídeo (Thrichomys sp.) e um caviídeo (Cavia aperea), exclusivamente capturadas nestas armadilhas (Tabela 2). Os pitfalls registraram a presença de 17 espécies na região, entre as quais um marsupial (Cryptonanus agricolai) e seis roedores cricetídeos (Calomys tener, Cerradomys scotti, Oecomys bicolor, Oligoryzomys fornesi, O. moojeni e Pseudoryzomys symplex) foram somente capturados nesse tipo de armadilha (Tabela 2). Os diferentes modelos de armadilhas de isca também apresentaram espécies exclusivas, os roedores Rhipidomys macrurus e Cavia aperea somente foram capturados em armadilhas Sherman, e o marsupial Didelphis albiventris somente em armadilhas gaiola. Com relação ao número de indivíduos capturados, o uso de armadilhas distintas também colaborou, dado que espécies de maior porte, como os roedores equimídeos, por exemplo, foram preferencialmente capturados nas gaiolas, provavelmente devido ao maior tamanho das mesmas. As estimativas de abundância destas espécies são melhor embasadas através do uso desta metodologia, que foi responsável por 64,6\% das capturas destes indivíduos na área de estudo (Tabela 2).

\subsection{Diversidade e abundância relativa}

As curvas de rarefação obtidas tanto para cada localidade quanto para o período total de amostragem apresentaram uma ascensão leve, porém contínua, ao longo do período de estudo (Figura 3), sugerindo que o número de espécies amostrado (23 - excluindo a única espécie exclusivamente amostrada manualmente, Caluromys lanatus) ainda é inferior ao número de espécies esperado para a região. A estimativa de riqueza obtida (28 espécies) (Figura 4) corrobora estes resultados. Entretanto, podemos considerar nossa amostragem satisfatória, dado que a maior parte desta comunidade foi amostrada no presente estudo (82\% da riqueza esperada).

A abundância relativa das espécies variou de $0,5 \%$ ( 1 indivíduo amostrado) a 27,3\% (56 indivíduos amostrados) (Tabela 2). Os marsupiais representaram $17,6 \%$ da comunidade, apresentando uma espécie de abundância mais elevada: Thylamys karimii $(11,2 \%)$ e cinco espécies raras: Cryptonanus agricolai (0,5\%), Didelphis albiventris $(1,0 \%)$, Gracilinanus agilis $(1,5 \%)$, Micoureus demerarae $(1,5 \%) \mathrm{e}$ Monodelphis domestica $(1,9 \%)$. Os roedores cricetídeos dominaram 
Carnignotto, A.P. \& Aires, C.C.

Tabela 2. Riqueza e abundância relativa (AR\%) das espécies de marsupiais e roedores amostradas por tipo de armadilha, $\mathrm{S}=\mathrm{Sherman}, \mathrm{G}=\mathrm{Gaiola}$ e $\mathrm{P}=$ pitfalls, na EESGT durante o período de estudo e em cada localidade (1- Mateiros, TO; 2- Rio da Conceição, TO e 3- Formosa do Rio Preto, BA).

Table 2. Richness and relative abundance (RA\%) of the marsupials and rodents surveyed by the live and pitfall traps, $\mathrm{S}=\mathrm{Sherman}, \mathrm{G}=$ wire and $\mathrm{P}=$ pitfall, in the EESGT during the study in each locality (1- Mateiros, TO; 2- Rio da Conceição, TO and 3- Formosa do Rio Preto, BA).

\begin{tabular}{|c|c|c|c|c|c|c|c|}
\hline Táxon & $\mathbf{P}$ & $\mathbf{S}$ & G & Total AR(\%) & $1 \operatorname{AR}(\%)$ & $2 \operatorname{AR}(\%)$ & $3 \operatorname{AR}(\%)$ \\
\hline \multicolumn{8}{|l|}{ Ordem Didelphimorphia } \\
\hline \multicolumn{8}{|l|}{ Família Didelphidae } \\
\hline Caluromys lanatus (Olfers, 1818)* & - & - & - & - & - & - & $01 *$ \\
\hline Cryptonanus agricolai (Moojen, 1943) & 01 & - & - & $01(0,5 \%)$ & - & $01(1,3 \%)$ & - \\
\hline Didelphis albiventris Lund, 1840* & - & - & 02 & $02(1,0 \%)$ & $01(1,3 \%)$ & $01 *$ & $01(1,9 \%)$ \\
\hline Gracilinanus agilis (Burmeister, 1854) & 02 & 01 & - & $03(1,5 \%)$ & - & $03(4,0 \%)$ & - \\
\hline Micoureus demerarae (Thomas, 1905) & - & 02 & 01 & $03(1,5 \%)$ & $01(1,3 \%)$ & $02(2,7 \%)$ & - \\
\hline Monodelphis domestica (Wagner, 1842) & 03 & 01 & - & $04(1,9 \%)$ & - & $03(4,0 \%)$ & $01(1,9 \%)$ \\
\hline Thylamys karimii (Petter, 1968) & 10 & 12 & 01 & $23(11,2 \%)$ & $10(12,8 \%)$ & $06(8,0 \%)$ & $07(13,5 \%)$ \\
\hline Total & 16 & 16 & 04 & $36(17,6 \%)$ & $12(15,4 \%)$ & $15(20 \%)$ & $09(17,3 \%)$ \\
\hline
\end{tabular}

Ordem Rodentia

Família Cricetidae

Calomys tener (Winge, 1887)

Cerradomys marinhus (Bonvicino, 2003)

Cerradomys scotti (Langguth \& Bonvicino, 2002)

Hylaeamys megacephalus (Fischer, 1814)

Necromys lasiurus (Lund, 1841)

Nectomys rattus (Pelzeln, 1883)

Oecomys bicolor (Tomes, 1860)

Oecomys paricola (Thomas, 1904)

Oligoryzomys fornesi (Massoia, 1973)*

Oligoryzomys moojeni Weksler \& Bonvicino, 2005

$\begin{array}{lcc}06 & - & - \\ 02 & - & 02 \\ 02 & - & - \\ 03 & - & 01 \\ 08 & 16 & 01 \\ - & 01 & 01\end{array}$

$06(2,9 \%)$

$-$

$03(4,0 \%)$

$03(5,8 \%)$

$04(1,9 \%)$

$-$

$01(1,3 \%)$

$03(5,8 \%)$

$02(1,0 \%)$

$04(1,9 \%)$

$01(1,3 \%)$

$01(1,3 \%)$

-

$25(12,2 \%)$

$05(6,4 \%)$

$04(5,3 \%)$

$-$

$02(1,0 \%)$

$04(1,9 \%)$

$\begin{array}{cccc}04 & - & - & 04(1,9 \%) \\ 02 & 02 & 01 & 05(2,4 \%)\end{array}$

$02-\quad-\quad-\quad 02(1,0 \%)$

$\begin{array}{cccc}01 & - & - & 01(0,5 \%) \\ 01 & 04 & 02 & 07(3,4 \%)\end{array}$

$-$

$06(8,0 \%)$

$14(26,9 \%)$

Oxymycterus delator Thomas, 1903

Pseudoryzomys simplex (Winge, 1887)

Rhipidomys macrurus (Gervais, 1855)*

$01(0,5 \%)$

01

$04-02$

-

$02(2,7)$

-

$03(3,8 \%)$

$04(5,3 \%)$

$02(2,5 \%) *$

$02(2,7 \%)$

$01(1,3 \%)$

$04(5,1 \%)$

$01(1,3 \%)$

$01 *$

$39(19,1 \%)$

$23(29,5 \%)$

$103(50,2 \%)$

$40(51,3 \%)$

Thalpomys cerradensis Hershkovitz, 1990

$21 \quad 15 \quad 03$

Família Caviidae

Cavia aperea Erxleben, 1777

- $01 \quad-\quad 01(0,5 \%)$

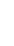

$01(0,5 \%)$

\begin{tabular}{|c|c|c|c|c|c|c|c|}
\hline Cavia aperea Erxleben, 1777 & - & 01 & - & $01(0,5 \%)$ & - & - & $01(1,9 \%)$ \\
\hline Total & - & 01 & - & $01(0,5 \%)$ & - & - & $01(1,9 \%)$ \\
\hline \multicolumn{8}{|l|}{ Família Echimyidae } \\
\hline Proechimys roberti Thomas, 1901 & 01 & 01 & 07 & $09(4,4 \%)$ & $08(10,3 \%)$ & $01(1,3 \%)$ & - \\
\hline Thrichomys sp.* & - & 21 & 35 & $56(27,3 \%)$ & $18(23,1 \%)$ & $18(24,0 \%) *$ & $20(38,5 \%)$ \\
\hline Total & 01 & 22 & 42 & $65(31,7 \%)$ & $26(33,3 \%)$ & $19(25,3 \%)$ & $20(38,5 \%)$ \\
\hline TOTAL & 70 & 78 & 57 & 205 & 78 & 75 & 52 \\
\hline
\end{tabular}

$01(1,9 \%)$

*01 espécime de Caluromys lanatus, 01 de Didelphis albiventris, 01 de Oligoryzomys fornesi, 01 de Rhipidomys macrurus e 01 de Thrichomys sp. foram amostrados manualmente.

a comunidade, abrangendo 50,2\% dos indivíduos capturados, sendo a tribo Akodontini a mais abundante (T. cerradensis - 19,1\%; $N$. lasiurus $-12,2 \%$ e $O$. delator - 3,4\%), representando $34,7 \%$ da comunidade, seguida pela tribo Oryzomini, que representou $12,6 \%$ dos indivíduos capturados (O. paricola $-2,4 \%$; C. marinhus $-1,9 \%$; H. megacephalus $-1,9 \%$; O. bicolor $-1,9 \%$; C. scotti $-1,0 \%$; $N$. rattus $-1,0 \%$; O. fornesi $-1,0 \% ; R$. macrurus $-1,0 \%$ e $O$. moojeni-0,5\%). A espécie de Phyllotini C. tener foi rara, sendo representada por seis indivíduos $(2,9 \%)$ e a espécie incertae sedis $P$. symplex ainda mais rara, com um único indivíduo capturado (0,5\%). A família Echimyidae foi representada por duas espécies
(Thrichomys sp.- 27,3\% e Proechimys roberti - 4,4\%), abrangendo $31,7 \%$ da comunidade amostrada (Tabela 2).

Com exceção das quatro espécies mais abundantes (Thrichomys sp., Thalpomys cerradensis, Necromys lasiurus e Thylamys karimii), que representaram $69,8 \%$ da comunidade amostrada, o restante das espécies esteve representada por um pequeno número de indivíduos (1 a 9), abrangendo 30,2\% da comunidade. Estes resultados, consequentemente, influenciaram o índice de equabilidade obtido $(E=0,46)$, que foi relativamente baixo. Da mesma forma, influenciaram o índice de diversidade calculado para a região $\left(H^{\prime}=2,36 ; D=10,59\right)$, que apesar disso apresentou uma riqueza alta 


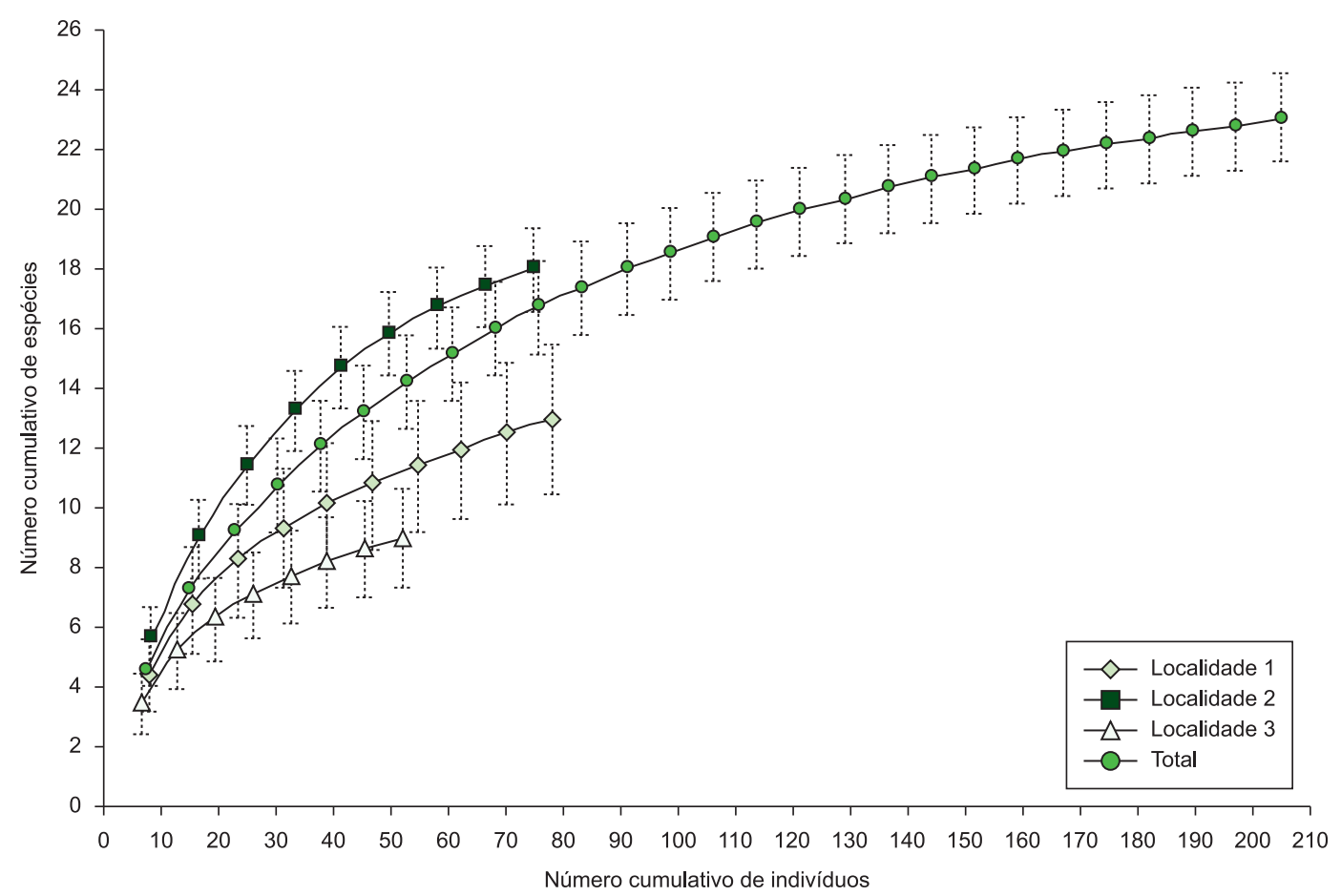

Figura 3. Curva média de incremento no número de espécies registradas com o aumento do esforço de amostragem empregado e do número de indivíduos amostrados em Mateiros, TO (localidade 1); Rio da Conceição, TO (localidade 2); Formosa do Rio Preto, BA (localidade 3); e ao longo de todo período de estudo na EESGT (Total). As barras representam os desvios padrão de cada valor médio.

Figure 3. Mean curve of the increased number of species registered with the increase in sampling effort and in the number of individuals surveyed in Mateiros, TO (locality 1); Rio da Conceição, TO (locality 2); Formosa do Rio Preto, BA (locality 3); and in the total period of the study in the EESGT (Total). The bars represent the standard deviation from each mean value.

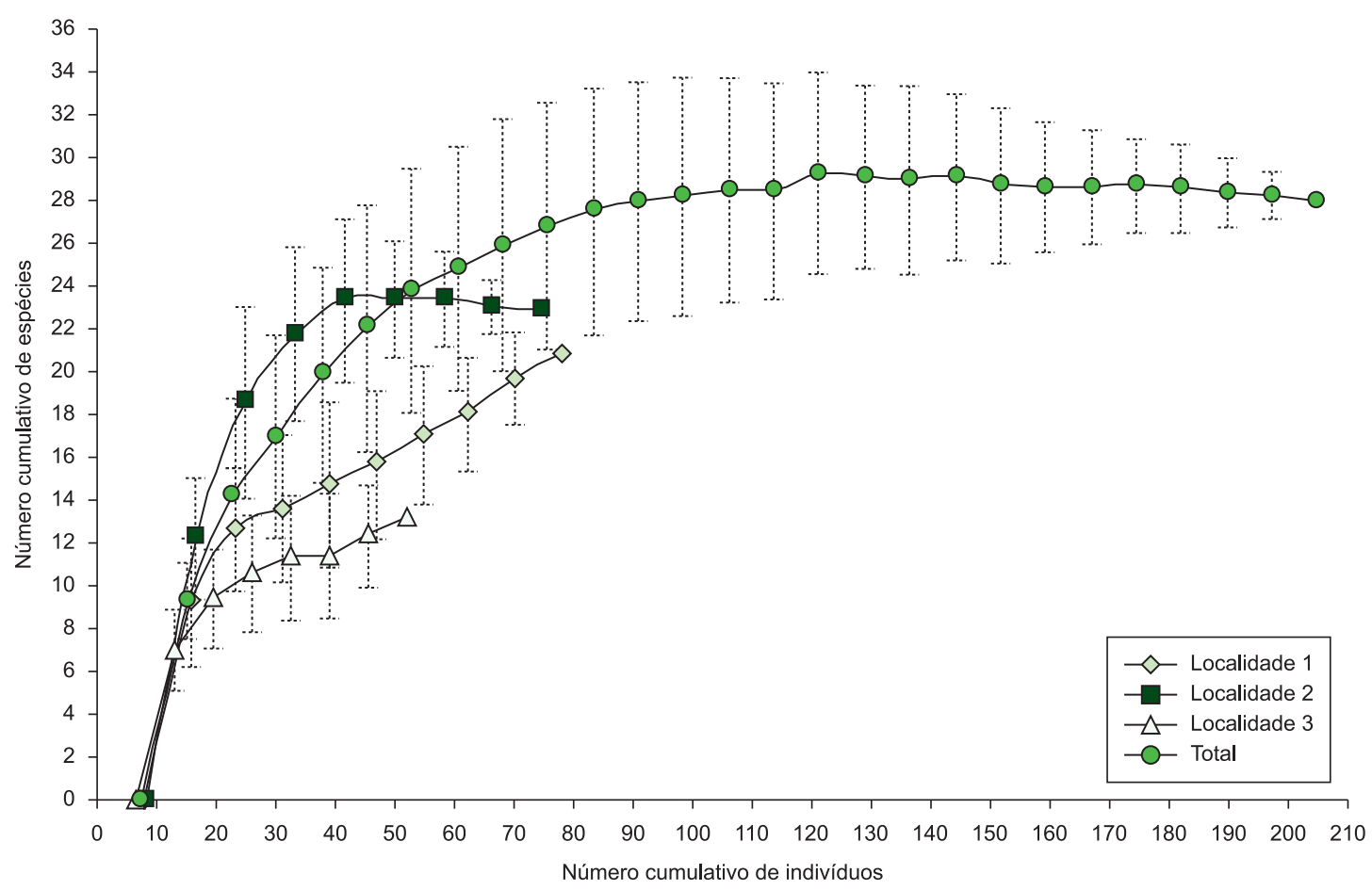

Figura 4. Curva média de incremento no número de espécies estimadas (Jackknife 2) com o aumento do esforço de amostragem empregado e do número de indivíduos amostrados em Mateiros, TO (localidade 1); Rio da Conceição, TO (localidade 2); Formosa do Rio Preto, BA (localidade 3); e ao longo de todo período de estudo na EESGT (Total). As barras representam os desvios padrão de cada valor médio.

Figure 4. Mean curve of the increased number of species estimated (Jackknife 2) with the increase in sampling effort and in the number of individuals surveyed in Mateiros, TO (locality 1); Rio da Conceição, TO (locality 2); Formosa do Rio Preto, BA (locality 3); and in the total period of the study in the EESGT (Total). The bars represent the standard deviation from each mean value. 
(23 espécies) (Tabela 3). Isoladamente, tanto o número de espécies como suas abundâncias contribuíram para a diversidade local, sendo a localidade mais rica (18 espécies) a que também apresentou uma maior equabilidade entre as espécies $(0,66)$ e, consequentemente, o maior índice de diversidade ( $\mathrm{H}^{\prime}=2,47$ - Tabela 3$)$.

Analisando cada uma das localidades separadamente, percebe-se que o padrão de abundância relativa se mantém nas três localidades amostradas, com os roedores cricetídeos sempre dominando, representando em torno de $50 \%$ da comunidade amostrada, seguidos dos roedores equimídeos, em torno de $30 \%$ e dos marsupiais, que representam em média $20 \%$ da comunidade (Tabela 2). A espécie de marsupial Thylamys karimii se manteve como a mais abundante nas três localidades amostradas, assim como o equimídeo Thrichomys sp. Em relação aos cricetídeos, Thalpomys cerradensis foi a espécie dominante em duas localidades, sendo substituída por Necromys lasiurus em uma das localidades amostradas. Apesar de espécies distintas, foram representantes da tribo Akodontini que dominaram as comunidades de pequenos mamíferos não voadores na região estudada (Tabela 2 ).

O número de espécies registrado em cada um dos hábitats amostrados variou de 5 a 9 (Tabela 4). Considerando a média de espécies registrada em cada um dos diferentes hábitats estudados, foi obtida uma diversidade alfa de 6,62 espécies $(28,8 \%$ da riqueza total). A diversidade beta (entre hábitats) foi de 16,38 espécies (71,2\% da riqueza total), dada a diversidade gama de 23 espécies registradas na área de estudo (Figura 5). Portanto, o número médio de espécies encontrado em cada um dos hábitats separadamente, foi 2,47 vezes menor do que a riqueza média encontrada, considerando-se todos os tipos de hábitats estudados. Nesta comunidade, portanto, a sobreposição de espécies entre os diferentes tipos de hábitats foi pequena, demonstrando que as espécies amostradas apresentam grande seletividade quanto às fisionomias onde ocorrem (Tabela 4), e que a diversidade da região é obtida à medida que a comunidade de cada uma destas fisionomias é amostrada (Figura 5).

Considerando-se a riqueza obtida em cada uma das localidades separadamente, a diversidade alfa foi de 13,33 espécies $(57,96 \%$ da riqueza total) (Tabela 3). A diversidade beta, ou seja, o número de espécies registrado entre as três localidades amostradas foi de 9,67 $(42,04 \%$ da riqueza total) (Figura 5), evidenciando que a maioria das espécies ocorreu em pelo menos duas das localidades amostradas, com algumas espécies (10) restritas a uma única localidade. Este dado revela a importância de amostrar não somente hábitats distintos, mas também regiões distintas, no sentido de obter estimativas mais confiáveis da riqueza presente em uma determinada área de estudo (Figura 5).

\subsection{Seletividade de hábitats}

Algumas espécies de marsupiais e roedores estiveram restritas a determinados tipos de hábitat. Como os dados de captura foram muito baixos para a maioria das espécies, em geral inferiores a cinco capturas, estes resultados não indicam necessariamente seletividade de hábitat. Estes dados também só podem ser comparados a partir da padronização do esforço de captura entre os hábitats (Tabela 4).

A comunidade de pequenos mamíferos não voadores foi composta de um marsupial, três roedores cricetídeos e um equimídeo restritos à formação florestal de mata de galeria (5 espécies - 21,7\%); quatro marsupiais, nove roedores cricetídeos, um caviídeo, e um equimídeo restritos aos ambientes abertos (15 espécies - 65,2\%); e um marsupial e dois cricetídeos que ocuparam ambos os tipos de fisionomias $(3-13,1 \%)$. Essa segregação foi identificada pela análise de correspondência não tendenciada (DCA), onde as espécies se dividiram basicamente em três grandes grupos ao longo do eixo 1 (autovalor $=0,945)($ Figura 6): o primeiro formado pelas espécies que ocorreram preferencialmente nas formações abertas de campo úmido, campo sujo, campo cerrado, cerrado sensu stricto e cerrado sensu stricto com afloramentos rochosos; o segundo representado por duas espécies capturadas exclusivamente em áreas de campo limpo; e o terceiro representado por espécies amostradas preferencialmente nas formações florestais. Considerando-se o eixo 2 desta mesma análise (autovalor $=0,441$ ), percebe-se, ainda, uma separação entre as duas formações florestais amostradas, com um grupo de espécies mais associado à mata de galeria, acima, e outro à mata de galeria úmida, abaixo (Figura 6). Estes resultados corroboram a análise de partição de riqueza realizada e apontam, novamente, para a importância dos diferentes hábitats, em especial dos dois tipos de fisionomias (florestal e aberta) para a riqueza específica de comunidades no domínio Cerrado.

\section{Mamíferos de médio e grande porte}

A fauna de mamíferos de médio e grande porte amostrada na região da EESGT foi composta de 17 gêneros e 17 espécies (Tabela 5). Lima et al. (2005) amostraram a fauna de mamíferos de médio e grande porte da região do Jalapão, TO, que abrange a Serra do Espírito Santo a partir do rio Novo e Mateiros, até o rio Come-Assado, onde se encontram representados os tipos de vegetação de cerrado sensu lato e mata de galeria. Os métodos de amostragem destes autores incluíram evidências diretas (visualização e atropelamento) e indiretas (rastros, fezes, pêlos e entrevista). Além das 17 espécies registradas para a EESGT, somam-se uma nova ordem (Pilosa), cinco famílias (Myrmecophagidae, Cebidae, Dasyproctidae, Erethizonthidae e Procyonidae) e 23 espécies de mamíferos de médio e grande porte (Tabela 5), totalizando 40 espécies para a região do Jalapão.

Há 10 espécies de mamíferos de médio e grande porte consideradas "Vulneráveis" (Brasil 2003) com ocorrência para a região do Jalapão (Tabela 5). Dentre estas, a Ordem Carnivora é a que apresenta o maior número de espécies ameaçadas (6 espécies): Leopardus pardalis, L. tigrinus, L. wiedii, Panthera onca, Chrysocyon brachyurus e Speothos venaticus; seguidas pelas ordens Cingulata (Priodontes maximus e Tolypeutes tricinctus), Pilosa (Myrmecophaga tridactyla) e Artiodactyla (Blastocerus dichotomus).

Tabela 3. Número de espécies obtido e esperado, suficiência em relação à amostragem, índice de diversidade (H') e índice de equabilidade (E) para cada uma das localidades amostradas e para a área de estudo.

Table 3. Number of sampled and estimated species, percentage of the survey, diversity index (H') and evenness index (E) for each locality and for the total area sampled.
Localidades
Riqueza obtida
Riqueza esperada
Suficiência da
Diversidade (H')
Equabilidade (E)

\begin{tabular}{llllll}
\hline Mateiros, TO & 13 & 20,79 & 62,53 & 2,02 & 0,58 \\
Rio da Conceição, TO & 18 & 22,93 & 78,50 & 2,47 & 0,66 \\
Formosa do Rio Preto, BA & 09 & 13,23 & 68,03 & 1,67 & 0,59 \\
\hline Total & 23 & 27,99 & 82,17 & 2,36 & 0,46 \\
\hline
\end{tabular}


Tabela 4. Dados de sucesso de captura (\%) das espécies de marsupiais e roedores nos diferentes hábitats amostrados durante o período de estudo na EESGT. $\mathrm{CU}=$ campo úmido e vereda; $\mathrm{CL}=$ campo limpo; $\mathrm{CS}=$ campo sujo $\mathrm{CC}=$ campo cerrado; $\mathrm{CSS}=$ cerrado sensu stricto $; \mathrm{CP}=$ cerrado com afloramentos rochosos, $\mathrm{MU}=$ mata úmida e $\mathrm{MG}=$ mata galeria .

Table 4. Capture success (\%) of the marsupials and rodent species in the different habitats surveyed during the study period in the EESGT. CU = campo úmido e vereda; $\mathrm{CL}=$ campo limpo; $\mathrm{CS}=$ campo sujo $\mathrm{CC}=$ campo cerrado; $\mathrm{CSS}=$ cerrado sensu stricto $; \mathrm{CP}=$ cerrado with rocks, $\mathrm{MU}=$ humid gallery forest and $\mathrm{MG}=$ gallery forest.

\begin{tabular}{|c|c|c|c|c|c|c|c|c|}
\hline Táxon & $\mathbf{C U}$ & CL & CS & $\mathrm{CC}$ & CSS & $\mathbf{C P}$ & MU & MG \\
\hline \multicolumn{9}{|l|}{ Ordem Didelphimorphia } \\
\hline \multicolumn{9}{|l|}{ Família Didelphidae } \\
\hline Cryptonanus agricolai & - & - & - & - & - & 0,10 & - & - \\
\hline Didelphis albiventris & - & - & - & - & - & 0,11 & - & 0,36 \\
\hline Gracilinanus agilis & - & - & - & - & 0,12 & 0,05 & - & - \\
\hline Micoureus demerarae & - & - & - & - & - & - & 1,67 & 0,36 \\
\hline Monodelphis domestica & - & - & - & - & - & 0,21 & - & - \\
\hline Thylamys karimii & - & 0,32 & 0,33 & 0,46 & 0,23 & 0,10 & - & - \\
\hline Total & - & 0,32 & 0,33 & 0,46 & 0,35 & 0,57 & 1,67 & 0,72 \\
\hline Riqueza & - & 01 & 01 & 01 & 02 & 05 & 01 & 02 \\
\hline \multicolumn{9}{|l|}{ Ordem Rodentia } \\
\hline \multicolumn{9}{|l|}{ Família Cricetidae } \\
\hline Calomys tener & $0,28 *$ & 0,08 & 0,33 & 0,29 & - & - & - & - \\
\hline Cerradomys marinhus & - & $0,14^{* *}$ & - & - & - & - & 0,36 & - \\
\hline Cerradomys scotti & - & - & - & 0,29 & 0,11 & - & - & - \\
\hline Hylaeamys megacephalus & - & - & - & - & - & - & 1,43 & - \\
\hline Necromys lasiurus & 0,62 & $0,18 * *$ & 0,22 & 0,74 & 0,06 & 0,05 & - & - \\
\hline Nectomys rattus & 0,26 & - & - & - & - & - & - & - \\
\hline Oecomys bicolor & - & - & - & - & - & - & 2,50 & - \\
\hline Oecomys paricola & - & - & - & - & - & - & 0,71 & 0,50 \\
\hline Oligoryzomys fornesi & - & - & 0,05 & - & - & 0,10 & - & - \\
\hline Oligoryzomys moojeni & - & $0,08 * *$ & - & - & - & - & - & - \\
\hline Oxymycterus delator & 0,44 & 0,05 & 0,05 & - & - & - & - & - \\
\hline Pseudoryzomys simplex & - & 0,08 & - & - & - & - & - & - \\
\hline Rhipidomys macrurus & - & - & - & - & 0,13 & - & - & - \\
\hline Thalpomys cerradensis & - & 0,55 & 0,82 & 0,93 & - & 0,10 & - & 0,17 \\
\hline Total & 1,60 & 1,16 & 1,47 & 2,25 & 0,30 & 0,25 & 5,00 & 0,67 \\
\hline Riqueza & 04 & 07 & 05 & 04 & 03 & 03 & 04 & 02 \\
\hline
\end{tabular}

Família Caviidae

\begin{tabular}{lccccccc} 
Cavia aperea & 0,13 & - & - & - & - & - & - \\
\hline Total & 0,13 & - & - & - & - & - & - \\
\hline Riqueza & 01 & - & - & - & - & - & - \\
\hline
\end{tabular}

\begin{tabular}{lcccccccc} 
Riqueza & 01 & - & - & - & - & - & - \\
\hline Família Echimyidae & & & & & & & & \\
Proechimys roberti & - & - & - & - & - & 0,36 & 1,34 \\
Thrichomys sp. & - & 0,22 & 0,22 & 2,32 & 1,18 & 2,60 & - & - \\
\hline Total & - & 0,22 & 0,22 & 2,32 & 1,18 & 2,60 & 0,36 & 1,34 \\
\hline Riqueza & - & 01 & 01 & 01 & 01 & 01 & 01 & 01 \\
\hline Total*** & 1,73 & 1,70 & 2,02 & 5,03 & 1,83 & 3,42 & 7,03 & 2,73 \\
\hline Riqueza & 05 & 09 & 07 & 06 & 06 & 09 & 06 & 05 \\
\hline
\end{tabular}

*Esta captura ocorreu em área de campo de murundum borda com o campo úmido.**Estas capturas ocorreram em área de campo limpo borda com o campo úmido. ***Os espécimes coletados manualmente foram capturados nos seguintes hábitats: Caluromys lanatus = dentro do rio Galhão, Didelphis albiventris = campo cerrado, Oligoryzomys fornesi e Rhipidomys macrurus = moradia e Thrichomys sp. = campo sujo. 


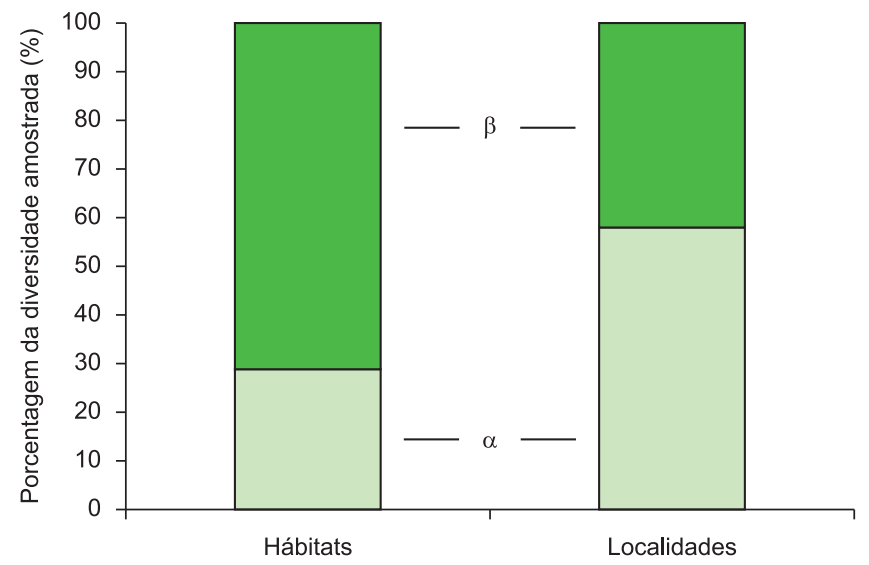

Figura 5. Partição aditiva da diversidade encontrada considerando-se a importância do mosaico de hábitats em cada localidade $(\alpha=$ riqueza em cada hábitat e $\beta=$ riqueza entre hábitats) e da localização geográfica para a amostragem total $(\alpha=$ riqueza em cada localidade e $\beta=$ riqueza entre localidades).

Figure 5. Diversity additive partitioning considering the importance of the habitat mosaic in each locality $(\alpha=$ richness in each habitat and $\beta=$ richness between habitats) and the geographic location for the whole area surveyed $(\alpha=$ richness in each locality and $\beta=$ richness between localities).

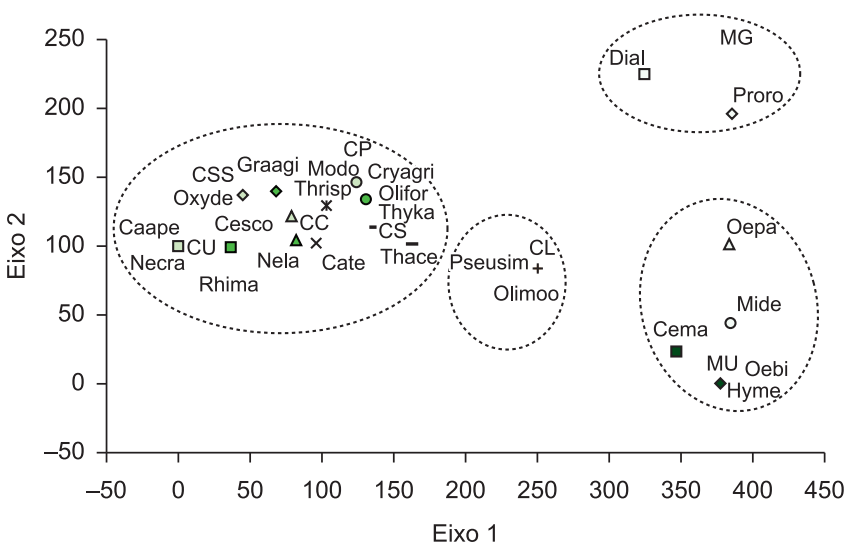

Figura 6. Escores das 23 espécies e dos oito hábitats amostrados nos dois primeiros eixos da análise de correspondência não tendenciada (DCA) baseados no sucesso de captura destas espécies nos hábitats amostrados. Os acrônimos referem-se à primeira sílaba do epíteto do gênero e do epíteto da espécie e em negrito as siglas dos hábitats amostrados ( $\mathrm{CU}=$ campo úmido e vereda; $\mathrm{CL}=$ campo limpo; $\mathrm{CS}=$ campo sujo; $\mathrm{CC}=$ campo cerrado; CSS $=$ cerrado sensu stricto $; \mathrm{CP}=$ cerrado com afloramentos rochosos, $\mathrm{MU}=$ mata úmida e $\mathrm{MG}=$ mata galeria) .

Figure 6. First and second axis DCA scores for the 23 species and the eight habitats surveyed based in the capture success of these species in the sampled habitats. The acronyms refer to the first genus syllabi and the first species syllabi and in bold letters to the habitats $(\mathrm{CU}=$ campo úmido e vereda; $\mathrm{CL}=$ campo limpo; $\mathrm{CS}=$ campo sujo $; \mathrm{CC}=$ campo cerrado $; \mathrm{CSS}=$ cerrado sensu stricto $; \mathrm{CP}=$ cerrado with rocks, $\mathrm{MU}=$ humid gallery forest and $\mathrm{MG}=$ gallery forest $)$.

\section{Discussão}

Estudos realizados no Cerrado (apresentando esforço mínimo de 1000 armadilhas.noite e mais de um tipo de hábitat amostrado) registraram entre 6 a 25 espécies de pequenos mamíferos não voadores (Marinho-Filho et al. 1994, Bonvicino et al. 1996, Talamoni \& Dias 1999, Lacher \& Alho 2001, Bonvicino et al. 2002a, Carmignotto 2005, Vieira \& Palma 2005, Cáceres et al. 2011), sendo a riqueza encontrada no presente estudo próxima aos valores máximos já registrados para o domínio. O número de gêneros (21) praticamente semelhante ao número de espécies (24) aqui obtido corrobora o padrão encontrado em outras comunidades de pequenos mamíferos do Cerrado (Vieira \& Palma 2005), indicando um padrão característico deste domínio. Este padrão é bastante interessante do ponto de vista biogeográfico dado que pode refletir a antiga diferenciação, representada pela elevada divergência filogenética entre as espécies, da fauna do Cerrado. Estudos a respeito da idade e endemismo da avifauna, por exemplo, corroboram esta hipótese (e.g. Silva 1995, Silva \& Bates 2002).

Apesar do sucesso de captura variar entre os diferentes tipos de armadilhas, em especial quando se compara trabalhos realizados em domínios distintos, os resultados aqui obtidos ressaltam o caráter complementar da metodologia utilizada, sendo essencial o uso de diferentes tipos e tamanhos de armadilhas na amostragem deste grupo de mamíferos (Lyra-Jorge \& Pivello 2001, Voss et al. 2001, Hice \& Schmidly 2002, Astúa et al. 2006, Umetsu et al. 2006). No caso das amostragens realizadas no Cerrado, as armadilhas convencionais ainda suplantam os pitfalls em relação ao sucesso de captura, fato que não ocorre na Mata Atlântica, onde o uso dos pitfalls vem se mostrando mais eficiente tanto na captura de espécies quanto de indivíduos (Lyra-Jorge \& Pivello 2001, Carmignotto 2005, Santos-Filho et al. 2006, Umetsu et al. 2006). O volume dos baldes utilizados parece influenciar sobremaneira estes resultados na medida em que baldes de volumes maiores (entre 60-100 L) apresentam sucesso de captura mais elevados quando comparados a baldes de 20-35 L (Cáceres et al. 2011). Não somente o uso de metodologia de amostragem complementar é importante para o registro de um maior número de espécies, mas também o esforço de amostragem, que no caso do Cerrado necessita ser relativamente alto, como os valores empregados neste e em outros inventários (e.g. Marinho-Filho et al. 1994, Carmignotto 2005, Vieira \& Palma 2005). Recomenda-se, portanto, o uso de tamanhos e tipos de armadilhas distintas (armadilhas de isca e armadilhas de queda) e um esforço mínimo em torno de 3000 armadilhas.noite para cada localidade, em inventários deste grupo de mamíferos no Cerrado.

Com relação à abundância relativa das espécies, o padrão obtido no presente estudo, de uma comunidade representada por poucas espécies abundantes e muitas espécies intermediárias e raras, assemelha-se ao padrão descrito por uma curva lognormal, geralmente encontrado em comunidades naturais, e caracterizado pela presença de poucas espécies abundantes, algumas espécies intermediárias e muitas espécies raras (Magurran 2004, Krebs 1999). O padrão lognormal de abundância já foi descrito para comunidades de pequenos mamíferos do Cerrado, sendo geralmente encontradas comunidades que apresentam valores intermediários de equabilidade, com algumas espécies abundantes, porém não apresentando valores muito discrepantes de abundância (Bonvicino et al. 1996, Lacher \& Alho 2001, Bonvicino et al. 2002a, Carmignotto 2005). Estes dados também refletem o estado de conservação do ambiente onde estas comunidades foram amostradas dado que em áreas alteradas, os valores de equabilidade são relativamente inferiores aos encontrados em áreas bem preservadas, apresentando espécies com elevado índice de abundância relativa (e.g. Bonvicino et al. 2002a, Carmignotto 2005). As localidades amostradas no presente estudo, portanto, podem ser classificadas como bem preservadas do ponto de vista da conservação do meio ambiente, baseado nos dados levantados para as comunidades de pequenos mamíferos.

Outro padrão observado neste trabalho diz respeito à seletividade de hábitats. A segregação pelas espécies amostradas entre as formações florestais e as áreas abertas é um padrão característico deste grupo faunístico no Cerrado (Bonvicino et al. 1996, Talamoni \& 
Tabela 5. Riqueza de espécies de mamíferos de médio e grande porte amostradas através de evidências diretas (visualização, captura, e animais encontrados mortos) e evidências indiretas (rastros, fezes e vocalização) na EESGT durante o período de estudo, e dados extraídos de Lima et al. (2005) para a região do Jalapão, TO.

Table 5. Large and medium size mammal species richness surveyed by direct (visual, capture, carcasses) and indirect (tracks, feces, vocalizations) in the EESGT during the study period and data extracted from Lima et al. (2005) for the Jalapão region in the Tocantins State.

\section{Táxon}

Ordem Cingulata

Família Dasypodidae

Cabassous unicinctus (Linnaeus, 1758)

Dasypus kappleri Krauss, 1862

Dasypus novemcinctus Linnaeus, 1758

Dasypus septemcinctus Linnaeus, 1758

Euphractus sexcinctus (Linnaeus, 1758)

Priodontes maximus (Kerr, 1792)*

Tolypeutes tricinctus (Linnaeus, 1758)*

Ordem Pilosa

Família Myrmecophagidae

Myrmecophaga tridactyla Linnaeus, 1758*

Tamandua tetradactyla (Linnaeus, 1758)

Ordem Primates

Família Cebidae

Callithrix penicillata (É. Geoffroy, 1812)

Cebus apella (Linnaeus, 1758)

Família Atelidae

Alouatta caraya (Humboldt, 1812)

Ordem Rodentia

Família Erethizontidae

Coendou prehensilis (Linnaeus, 1758)

Família Caviidae

Hydrochoerus hydrochaeris (Linnaeus, 1766)

Família Dasyproctidae

Dasyprocta leporina (Linnaeus, 1758)

Família Cuniculidae

Cuniculus paca (Linnaeus, 1766)

Ordem Carnivora

Família Felidae

Leopardus pardalis (Linnaeus, 1758)*

Leopardus tigrinus (Schreber, 1775)*

Leopardus wiedii (Schinz, 1821)*

Panthera onca (Linnaeus, 1758)*

Puma concolor (Linnaeus, 1771)

Puma yagouaroundi (É. Geoffroy, 1803)

Família Canidae

Atelocynus microtis (Sclater, 1883)

Cerdocyon thous (Linnaeus, 1766)

Chrysocyon brachyurus (Illiger, 1815)*

Lycalopex vetulus (Lund, 1842)

Speothos venaticus (Lund, 1842)*

Família Mustelidae

Eira barbara (Linnaeus, 1758)

Galictis vittata (Schreber, 1776)

Lontra longicaudis (Olfers, 1818)

\section{Tipo de Registro}

EESGT, TO

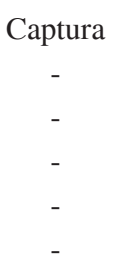

Captura

$$
-
$$$$
-
$$

$-$

$-$

Captura

-

Fezes

-

Visualização

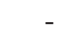

$-$

$-$

Rastros

-

Visualização

Rastros e Vocalização

Visualização

Visualização
Tipo de Registro Jalapão, TO
Atropelamento

Atropelamento

Visualização

Visualização

Visualização

Pêlo/Entrevista

Visualização

Fezes/Entrevista

Visualização

Visualização

Atropelamento

Atropelamento

Rastros/Entrevista

Visualização

Atropelamento

Visualização

Visualização

Visualização

Visualização

Visualização

Visualização

Visualização

* Espécies ameaçadas de extinção segundo "Lista Nacional das Espécies da Fauna Brasileira Ameaçadas de Extinção" publicada pelo MMA (Brasil 2003). 
Carnignotto, A.P. \& Aires, C.C.

\begin{tabular}{|c|c|c|}
\hline Táxon & $\begin{array}{l}\text { Tipo de Registro } \\
\text { EESGT, TO }\end{array}$ & $\begin{array}{c}\text { Tipo de Registro } \\
\text { Jalapão, TO }\end{array}$ \\
\hline \multicolumn{3}{|l|}{ Família Mephitidae } \\
\hline Conepatus semistriatus (Boddaert, 1785) & Visualização & Visualização \\
\hline \multicolumn{3}{|l|}{ Família Procyonidae } \\
\hline Nasua nasua (Linnaeus, 1766) & - & Visualização \\
\hline Procyon cancrivorus (G. Cuvier, 1798) & - & Visualização \\
\hline \multicolumn{3}{|l|}{ Família Tapiridae } \\
\hline Tapirus terrestris (Linnaeus, 1758) & Rastros e Esqueleto & Visualização \\
\hline \multicolumn{3}{|l|}{ Ordem Artiodactyla } \\
\hline \multicolumn{3}{|l|}{ Família Tayassuidae } \\
\hline Pecari tajacu (Linnaeus, 1758) & - & Visualização \\
\hline Mazama americana (Erxleben, 1777) & - & Visualização \\
\hline Mazama gouazoubira (Fischer, 1814) & $\begin{array}{c}\text { Fezes, Rastros, Visualização e } \\
\text { Esqueleto }\end{array}$ & Visualização \\
\hline Ozotoceros bezoarticus (Linnaeus, 1758) & Visualização & - \\
\hline
\end{tabular}

Dias 1999, Lacher \& Alho 2001, Bonvicino et al. 2002a, Carmignotto 2005). Análises de similaridade faunística entre hábitats ao longo deste domínio têm demonstrado que a composição de pequenos mamíferos das áreas abertas difere da composição encontrada em ambientes florestais, dividindo a comunidade em dois grupos distintos (Bonvicino et al. 1996, Lacher \& Alho 2001, Carmignotto 2005). No presente estudo ressalta-se, ainda, a importância da representatividade dos tipos de hábitats na paisagem. Como na EESGT as áreas abertas predominam, um maior número de espécies esteve associado a este tipo fisionômico. Os resultados de abundância relativa também corroboram este padrão dado que as espécies mais abundantes são espécies estreitamente relacionadas aos hábitats abertos (campo limpo, campo sujo e cerrado s. s.). Os inventários no Cerrado devem abranger a heterogeneidade ambiental presente nas localidades amostradas para que espécies especialistas florestais, especialistas das formações abertas, e generalistas sejam registradas.

Em termos biogeográficos, a fauna registrada na EESGT apresenta grande sobreposição com a fauna encontrada no domínio da Caatinga, representada pelas espécies que ocorrem em ambos os domínios, como por exemplo, os marsupiais Didelphis albiventris, Gracilinanus agilis, Monodelphis domestica e Thylamys karimii; e os roedores Calomys tener, Necromys lasiurus, Oligoryzomys moojeni, Pseudoryzomys simplex e Rhipidomys macrurus (Oliveira et al. 2004). No entanto, também apresenta espécies que se distribuem no domínio Amazônico, como os marsupiais Caluromys lanatus e Micoureus demerarae; e os roedores Hylaeamys megacephalus, Nectomys rattus, Oecomys bicolor, O. paricola e Proechimys roberti (Voss et al. 2001, Costa 2003), evidenciando que há uma afinidade faunística com a Amazônia para as espécies que habitam ambientes florestais, e com a Caatinga para as espécies que ocorrem nas áreas abertas. Além disso, as espécies registradas na EESGT podem ser classificadas em dois grupos: - as amplamente distribuídas no Cerrado (15), como é o caso de Caluromys lanatus, Cryptonanus agricolai, Didelphis albiventris, Gracilinanus agilis, Monodelphis domestica, Calomys tener, Cerradomys scotti, Hylaeamys megacephalus, Necromys lasiurus, Oligoryzomys fornesi,
Oecomys bicolor, Oxymycterus delator, Rhipidomys macrurus, Cavia aperea e Proechimys roberti; e - as espécies que apresentam distribuição restrita neste domínio (9), como Micoureus demerarae, Thylamys karimii, Cerradomys marinhus, Nectomys rattus, Oecomys paricola, Oligoryzomys moojeni, Pseudoryzomys simplex, Thalpomys cerradensis e Thrichomys sp. Estas últimas distribuemse, principalmente, nas porções norte e nordeste do Cerrado, revelando uma diferenciação regional ao longo da fauna de pequenos mamíferos deste domínio (Carmignotto 2005). Além disso, há as espécies endêmicas do Cerrado que ocorrem na EESGT e que estão representadas pelos roedores Cerradomys marinhus, Oligoryzomys moojeni, Thalpomys cerradensis e Thrichomys sp., todos com distribuição restrita à porção norte do Cerrado (Carmignotto 2005). Apesar da fauna da EESGT apresentar mais espécies compartilhadas com outros biomas do que espécies que ocorrem exclusivamente no Cerrado, ela representa uma porção distinta dentro do Cerrado, devido justamente à ocorrência de espécies compartilhadas com a Amazônia, com a Caatinga, e das endêmicas restritas à porção norte do Cerrado.

Estes padrões são dificilmente observados nas comunidades de mamíferos de médio e grande porte dado que a maioria das espécies deste grupo apresenta ampla distribuição geográfica, ocorrendo em praticamente todo o território brasileiro. No entanto, a ocorrência de algumas espécies deste grupo denota a forte influência Amazônica nesta região, dado que a espécie de tatu Dasypus kappleri, a espécie de cutia Dasyprocta leporina, e a espécie de cachorro-do-mato Atelocynus microtis apresentam a maior parte de sua distribuição restrita a este domínio. Entretanto, estas espécies somente foram registradas com base em entrevistas e visualização, não havendo material testemunho (Lima et al. 2005), sendo importante a confirmação destes registros para a região do Jalapão.

Com relação à conservação das espécies presentes na EESGT, a ocorrência de populações do cervo-do-Pantanal (Blastocerus dichotomus) nesta região é de extrema importância, dado que esta espécie apresenta distribuição geográfica disjunta, sendo representada por populações isoladas. Como existem grandes 
extensões de veredas no interior da EESGT, um dos ambientes favoritos desta espécie, é provável que estas abriguem populações de cervo-do-Pantanal, sendo extremamente importante um estudo mais detalhado da densidade populacional e monitoramento das populações aí presentes. Ressalta-se, ainda, a presença de Lycalopex vetulus, a raposinha, antes considerada espécie endêmica do Cerrado (MarinhoFilho et al. 2002), mas que atualmente já foi registrada em áreas de Caatinga no sudoeste da Bahia (A. P. Carmignotto, obs. pess.). Considerando-se as espécies ameaçadas de extinção, dez espécies de mamíferos de médio e grande porte consideradas "Vulneráveis" (Brasil 2003) ocorrem na região do Jalapão, aumentando ainda mais a importância desta região para a conservação das espécies de mamíferos brasileiras.

\section{A Fauna de Mamíferos da Estação Ecológica Serra Geral do Tocantins}

No presente estudo foram amostradas 24 espécies de pequenos mamíferos não voadores e 17 espécies de mamíferos de médio e grande porte, totalizando 41 espécies de mamíferos para as localidades de Mateiros, TO; Rio da Conceição, TO e Formosa do Rio Preto, BA inseridas na EESGT. Se considerarmos que os mamíferos de médio e grande porte não tiveram uma amostragem sistematizada como os pequenos, é esperado que este número seja incrementado, dado que para a região do Jalapão, Lima et al. (2005) amostraram outras 23 espécies. Estes resultados evidenciam a elevada riqueza de espécies de mamíferos presente na região quando comparada a outros inventários realizados no Cerrado.

Considerando-se os pequenos mamíferos, a comunidade foi representada, em geral, por várias espécies raras ou de abundância intermediária, e poucas espécies muito abundantes. Os roedores cricetídeos dominaram tanto em número de espécies (14) quanto em abundância (50,2\% da comunidade). As espécies de marsupiais e roedores amostradas se dividiram em dois grupos distintos: 1- espécies que ocorreram apenas nos ambientes florestais; e 2- espécies exclusivas dos ambientes abertos, demonstrando a grande seletividade de hábitats e a importância de se amostrar o mosaico de hábitats presente na região para uma melhor caracterização da diversidade desta comunidade. Em termos biogeográficos, a comunidade apresentou sobreposição com a fauna da Caatinga ( 9 espécies compartilhadas) e da Amazônia (7 espécies compartilhadas), evidenciando a importância destes domínios para a composição de espécies presente nesta porção norte do Cerrado; além da presença de espécies endêmicas (4 espécies).

A fauna de mamíferos da EESGT não somente é importante devido ao número de espécies que foram ali registradas, mas também devido à composição de espécies que abriga, incluindo, além das espécies compartilhadas com os domínios adjacentes, espécies endêmicas, e que apresentam distribuição restrita dentro do Cerrado, sendo extremamente importante sua preservação. Dentre as quatro espécies mais abundantes, constam espécies pouco conhecidas, como Thylamys karimii, que teve seu status taxonômico revisado, sendo considerada uma espécie válida apenas recentemente (Carmignotto \& Monfort 2006) e Thrichomys sp., táxon provavelmente distinto das outras espécies do gênero (Basile 2003, Braggio \& Bonvicino 2004), ressaltando a necessidade de estudos nesta região do Cerrado. Além disso, há também a presença de espécies ameaçadas de extinção, que têm nesta área aumentadas as suas chances de sobrevivência.

\section{Agradecimentos}

Gostaríamos de agradecer à Fundação O Boticário de Proteção à Natureza, à Pequi - Pesquisa e Conservação do Cerrado, à Conservação Internacional do Brasil, ao projeto BIOTA FAPESP
(98/05075-7), à FAPESP (00/06642-4) e ao Museu de Zoologia da Universidade de São Paulo (MZUSP) pelo apoio financeiro e logístico para a realização das excursões de campo para a EESGT. Ao IBAMA e ICMBio pelas licenças de captura, coleta e transporte das espécies de mamíferos amostradas (Licenças número 150/2002 e 12187). À Prefeitura da cidade de Rio da Conceição pelo apoio logístico. Ao taxidermista Paulo César Balduino e sua estagiária Tainá pela preparação dos espécimes coletados. Ao professor Fernando J. N. Villela e aos alunos de graduação da UFSCar Campus Sorocaba: Caroline de Bianchi Tocchet, Débora dos Santos Mota, Igor Bhering da Costa e Rafael Zanelli pelo auxílio no trabalho de campo e na triagem do material científico. E em especial a Cristiano C. Nogueira pelo convite para participarmos do projeto "Inventário e Zoneamento de Vertebrados da Estação Ecológica Serra Geral do Tocantins: subsídios ao Plano de Manejo" e deste volume especial da revista Biota Neotropica.

\section{Referências Bibliográficas}

ASTÚA, D, MOURA, R.T., GRELLE, C.E.V. \& FONSECA, M.T. 2006. Influence of baits, trap type and position for small mammal capture in a Brazilian lowland Atlantic Forest. Bol. Mus. Biol. Mello Leitão 19: 31-44.

BASILE, P. 2003. Taxonomia de Thrichomys Trouessart, 1880 (Rodentia, Echimyidae). Dissertação de Mestrado. Departamento de Biologia, Faculdade de Filosofia, Ciências e Letras de Ribeirão Preto da Universidade de São Paulo.

BECKER, M. \& DALPONTE, J.C. 1991. Rastros de mamíferos silvestres brasileiros - um guia de campo. Editora Universidade de Brasília, Brasília.

BONVICINO, C.R. 2003. A new species of Oryzomys (Rodentia, Sigmodontinae) of the subflavus group from the Cerrado of Central Brazil. Mammalian Biology 68: 78-90.

BONVICINO, C.R., CERQUEIRA, R. \&. SOARES, V.A. 1996. Habitat use by small mammals of upper Araguaia river. Revista Brasileira de Biologia 56: 761-767.

BONVICINO, C.R., LINDBERGH, S.M. \& MAROJA, L.S. 2002a. Small non-flying mammals from conserved and altered areas of Atlantic forest and Cerrado: comments on their potential use for monitoring environment. Braz. J. Biol. 62(4B):765-774.

BONVICINO, C.R., OTAZU, I. \& D’ÁNDREA, P.S. 2002b. Karyologic evidence of diversification of the genus Thrichomys (Rodentia, Echimyidae). Cytogenet. Genome Res. 97:200-204. PMid:12438714. doi:10.1159/000066613

BORGES, P.A.L. \& TOMÁS, W.M. 2004. Guia de rastros e outros vestígios de mamíferos do Pantanal. Embrapa Pantanal, Corumbá.

BRAGGIO, E. \& BONVICINO, C.R. 2004. Molecular divergence in the genus Thrichomys (Rodentia, Echimyidae). J. Mammal. 85(2):316-320. doi:10.1644/1545-1542(2004)085<0316:MDITGT>2.0.CO;2

BRASIL. Ministério do Meio Ambiente - MMA. 2003. Instrução normativa № 3, de 27 de maio de 2003. DOU, Brasília, DF. Seção 1 101:88-97.

BROSE, U., MARTINEZ, N.D. \& WILLIAMS, R.J. 2003. Estimating species richness: sensitivity to sample coverage and insensitivity to spatial patterns. Ecology 84(9):2364-2377. doi:10.1890/02-0558

CÁCERES, N.C, NÁPOLI, R.P. \& HANNIBAL, W. 2011. Differential trapping success for small mammals using pitfall and standard cage traps in a woodland savannah region of southwestern Brazil. Mammalia 75:45-52. doi:10.1515/MAMM.2010.069

CARMIGNOTTO, A.P. 2005. Pequenos mamíferos terrestres do bioma Cerrado: padrões faunísticos locais e regionais. Tese de doutorado, Instituto de Biociências, Universidade de São Paulo, São Paulo.

CARMIGNOTTO, A.P. \& MONFORT, T. 2006. Taxonomy and distribution of the Brazilian species of Thylamys (Didelphimorphia: Didelphidae). Mammalia 70(1/2):126-144. 
CAVALCANTI, R.B.C. \& JOLY, A. 2002. Biodiversity and Conservation Priorities in the Cerrado Region. In The Cerrados of Central Brazil. (P.S. Oliveira \& R.J. Marquis, eds.). Columbia University Press, New York, p. 351-367.

CECHIN, S.Z \& MARTINS, M. 2000. Eficiência de armadilhas de queda (pitfall traps) em amostragens de anfíbios e répteis no Brasil. Rev. Bras. Zool. 17(3):729-740.

COLLI, G.R., BASTOS, R.P. \& ARAÚJO, A.F.B. 2002. The character and dynamics of the Cerrado herpetofauna. In The Cerrados of Brazil (P.S. Oliveira \& R.J. Marquis, eds.). Columbia University Press, New York, p. 223-241.

COLWELL, R.K. 2004. EstimateS: Statistical estimation of species richness and shared species from samples. (Software and User's Guide). Version 8. http://viceroy.eeb.uconn.edu/ estimates.

COSTA, G.C., NOGUEIRA, C., MACHADO, R.B. \& COLLI, G.R. 2007. Squamate richness in the Brazilian Cerrado and its environmentalclimatic associations. Divers. Distrib. 13:714-724. doi:10.1111/j.14724642.2007.00369.x

COSTA, L.P. 2003. The historical bridge between the Amazon and the Atlantic forests of Brazil: a study of molecular phylogeography with small mammals. J. Biogeogr. 30:71-86. doi:10.1046/j.1365-2699.2003.00792.x

EISENBERG, J.F. \& REDFORD, K.H. 1999. Mammals of the Neotropics - the central neotropics. University of Chicago Press, Chicago. v. 3.

EITEN, G. 1994. Vegetação do Cerrado. In Cerrado: caracterização, ocupação e perspectivas (M.N. Pinto, org.). Editora Universidade de Brasília, Brasília, p. 17-73.

EMMONS, L.H. \& FEER, F. 1997. Neotropical rainforest mammals - A field guide. Second edition. University of Chicago Press, Chicago, $307 \mathrm{p}$.

GONÇALVES, P.R., ALMEIDA, F.C. \& BONVICINO, C.R. 2005. A new species of Wiedomys (Rodentia: Sigmodontinae) from Brazilian Cerrado. Mamm. Biol. 70(1):46-60.

GOTELLI, N.J. \& COLWELL, R.K. 2001. Quantifying biodiversity: procedures and pitfalls in the measurement and comparison of species richness. Ecol. Lett. 4:379-391. doi:10.1046/j.1461-0248.2001.00230.x

HERSHKOVITZ, P. 1990a. The Brazilian rodent genus Thalpomys (Sigmodontinae, Cricetidae) with a description of a new species. J. Nat. H. 24:763-783.

HERSHKOVITZ, P. 1990b. Mice of the Akodon boliviensis size class (Sigmodontinae, Cricetidae), with the description of two new species from Brazil. Fieldiana, Zool. 57:1-35.

HERSHKOVITZ, P. 1993. A new Central Brazilian genus and species of sigmodontine rodent (Sigmodontinae) transitional between akodonts and oryzomyines, with a discussion of muroid molar morphology and evolution. Fieldiana, Zool. 75:1-18.

HICE, C.L. \& SCHMIDLY, D.J. 2002. The effectiveness of pitfall traps for sampling small mammals in the Amazon basin. Mastozool. Neotrop. / J. Neotrop. Mammal. 9(1):85-89.

INSTITUTO BRASILEIRO DO MEIO AMBIENTE E DOS RECURSOS NATURAIS RENOVÁVEIS - IBAMA. 2004. Unidade: Estação Ecológica Serra Geral do Tocantins/TO/BA. http://www2.ibama.gov.br/unidades/ estacoes/reuc/149.htm (último acesso em 15/07/2009)

INSTITUTO BRASILEIRO DE GEOGRAFIA E ESTATÍSTICA - IBGE. 1993. Mapa de Vegetação do Brasil. $2^{\text {nd }}$ ed. Escala 1: 5.000.000. IBGE.

KLINK, C.A. \& MACHADO, R.B. 2005. Conservation of the Brazilian Cerrado. Conserv. Biol. 19:707-713. doi:10.1111/j.1523-1739.2005.00702.x

KREBS, C.J. 1999. Ecological Methodology. Second Edition. Addison Welsey Educational Publishers, Inc., Menlo Park, Califórnia.

LACHER, T.E.J. \& ALHO, C.J.R. 2001. Terrestrial small mammal richness and habitat associations in an Amazon Forest-Cerrado Contact Zone. Biotropica 33(1):171-181.

LANGGUTH, A. \& C.R. BONVICINO. 2002. The Oryzomys subflavus species group, with description of two new species (Rodentia, Muridae, Sigmodontinae). Arquivos do Museu Nacional, Rio de Janeiro 60 (4): 285-294.
LEEMANS, R. \& CRAMER, W.P. 1991. The IIASA database for mean monthly values of temperature, precipitation, and cloudiness on a global terrestrial grid. RR-91-18. International Institute for Applied Systems Analysis, Laxenburg.

LIMA, J.F.S., HIDASI, J. \& VEIGA, N. 2005. Estudo da diversidade de mamíferos de médio e grande porte da região do Jalapão, Tocantins, Brasil. Bol. Mus. Para. Emilio Goeldi. Ser. Cienc. Nat. 1(2):233-240.

LOREAU, M. 2000. Are communities saturated? On the relationship between alpha, beta, and gamma diversity. Ecol. Lett. 3:73-76. doi:10.1046/j.14610248.2000.00127.x

LYRA-JORGE, M.C. \& PIVELLO, V.R. 2001. Combining live trap and pitfall to survey terrestrial small mammals in savanna and forest habitats, in Brazil. Mammalia 65(4):524-530.

MAGURRAN, A.E. 2004. Measuring biological diversity. Oxford: Blackwell Publishing.

MAMEDE, F., GARCIA, P.Q. \& SOUSA JÚNIOR, W.C.. 2002. Análise da viabilidade sócio-econômico-ambiental da transposição de águas da bacia do rio Tocantins para o rio São Francisco na região do Jalapão/ TO. Relatório Final. http://www.conservation-strategy.org/Reports/ pro_texto_final.pdf (último acesso em 15/07/2009).

MARINHO-FILHO, J., REIS, M.L., OLIVEIRA, P.S., VIEIRA, E.M. \& PAES, M.N. 1994. Diversity standards and small mammal numbers: conservation of the Cerrado biodiversity. An. Acad. Bras. Cienc. 66:149-157.

MARINHO-FILHO, J., RODRIGUES, F.H.G. \& JUAREZ, K.M. 2002. The Cerrado mammals: diversity, ecology, and natural history. In The Cerrados of Brazil (Oliveira, P.S. \& Marquis, R.J., eds.). Columbia University Press, New York, p. 266-286.

MARRIS, E. 2005. The forgotten ecossystem. Nature 437(13):944-945. PMid:16222267. doi:10.1038/437944a

McCUNE, B. \& MEFFORD, M.J. 1999. Multivariate analysis of ecological data. Version 4.25. MjM Software, Gleneden Beach, Oregon.

MOOJEN, J. 1943. Captura e preparação de pequenos mamíferos para coleções de estudos. Imprensa Nacional, Rio de Janeiro.

MYERS, N., MITTERMEIER, R.A., MITTERMEIER, C.G., FONSECA, G.A.B. \& KENT, J. 2000. Biodiversity hotspots for conservation priorities. Nature 403:853-858. PMid:10706275. doi:10.1038/35002501

OLIVEIRA, P.S. \& MARQUIS, R.J. 2002. Introduction: Development of research in the Cerrados. In The Cerrados of Brazil (P.S. Oliveira \& R.J. Marquis, eds.). Columbia University Press, New York, p. 1-12.

OLIVEIRA, A.T. \& RATTER, J.A. 2002. Vegetation physiognomies and woody flora of the Cerrado biome. In The Cerrados of Brazil (P.S. Oliveira \& R.J. Marquis, eds.). Columbia University Press, New York. p 91-120.

OLIVEIRA, J.A., GONÇALVES, P.R. \& BONVICINO, C.R. 2004. Mamíferos da Caatinga. In Ecologia e Conservação da Caatinga (I.R. Leal, M. Tabarelli \& J.M.C. Silva). Editora da Universidade Federal de Pernambuco, p. 275-302.

PALMER, M.W. 2004. Ordination Methods - an overview. University of Toronto Press, Toronto.

PATRIC, E.F. 1970. Bait preference of small mammals. J. Mammal. 51(1):179-182. doi:10.2307/1378558

SANTOS-FILHO, M., SILVA, D.J. \& SANAIOTTI, T.M. 2006. Efficiency of four trap types in sampling small mammals in forest fragments, Mato Grosso, Brazil. Mastozool. Neotrop. 13:217-225.

SILVA, J.M.C. 1995. Biogeographic analysis of the South American Cerrado avifauna. Steenstrupia 21: 49-67.

SILVA, J.M.C. \& BATES, J.M. 2002. Biogeographic patterns and conservation in the South American Cerrado: a tropical savanna hotspot. Bioscience 52(3): 225-233.

SMITH, M.F. \& PATTON, J.L. 1999. Phylogenetic relationships and the radiation of sigmodontine rodents in South America: evidence from cytochrome $b$. J. Mamm. Evol. 6(2):89-128. doi:10.1023/A:1020668004578 
TALAMONI, S.A. \& DIAS, M.M. 1999. Population and community ecology of small mammals in southeastern Brazil. Mammalia 63(2):167-181. doi:10.1515/mamm.1999.63.2.167

UMETSU F, NAXARA, L \& PARDINI, R. 2006. Evaluating the efficiency of pitfall traps for sampling small mammals in the Neotropics. J. Mammal. 87:757-765 doi:10.1644/05-MAMM-A-285R2.1

URBAN, D.I. 2000. Multivariate Analysis in Ecology. University of Toronto Press, Toronto.

VEECH, J.A., SUMMERVILLE, K.S., CRIST, T.O. \& GERING, J.C. 2002. The additive partitioning of species diversity: recent revival of an old idea. Oikos 99:3-9. doi:10.1034/j.1600-0706.2002.990101.x

VIEIRA, E.M. \& PALMA, A.R.T. 2005. Pequenos mamíferos do Cerrado: distribuição dos gêneros e estrutura das comunidades nos diferentes hábitats. In Cerrado: ecologia, biodiversidade e conservação (A. Scariot, J.M Felfili \& J.C. Sousa-Silva, eds.). Ministério do Meio Ambiente, Brasília, p. 265-282.

VILLELA, F.N.J. \& C. NOGUEIRA. Este volume. Geology and Geomorphology of Serra Geral do Tocantins Ecological Station. Biota Neotrop.

VOSS, R.S. \& EMMONS, L.H. 1996. Mammalian diversity in Neotropical lowland rainforests: a preliminary assessment. Bull. Am. Mus. Nat. Hist. 230:1-115.

VOSS, R.S., LUNDE, D.P. \& JANSA, S.A. 2005. On the contents of Gracilinanus Gardner and Creighton, 1989, with the description of a previously unrecognized clade of small didelphid marsupials. American Museum Novitates 3482: 1-34.
VOSS, R.S., LUNDE, D.P. \& SIMMONS, N.B. 2001. The mammals of Paracou, French Guiana: a neotropical lowland rainforest fauna. Part 2. nonvolant species. Bull. Am. Mus. Nat. Hist. 263:1-236.

WALTER, H. 1986. Vegetação e zonas climáticas: tratado de ecologia global. EPU, São Paulo.

WEKSLER., M. \& BONVICINO, C.R. 2005. Taxonomy of pigmy rice rats (genus Oligoryzomys, Rodentia: Sigmodontinae) of the Brazilian Cerrado, with the description of two new species. Arq. Mus. Nac., Rio J. 63(1):113-130

WEKSLER., M., PERCEQUILLO, A.R \& VOSS, R.S.. 2006. Ten new genera of Oryzomyine rodents (Cricetidae: Sigmodontinae). Am. Mus. Novit. 3537:1-29. doi:10.1206/0003-0082(2006)3537[1:TNGOOR]2.0.CO;2

WHITTAKER, R.H. 1972. Evolution and measurement of species diversity. Taxon 21:213-251. doi:10.2307/1218190

WILSON, D.E. \& REEDER, D.M. 2005. Mammal species of the world - a taxonomic and geographic reference. $3^{\text {nd }}$ ed. The John Hopkins University Press, Baltimore.

WILSON, D.E., COLE, F.R., NICHOLS, J.D., RUDRAN, R. \& FOSTER, M.S. 1996. Measuring and monitoring biological diversity - Standard methods for mammals. Smithsonian Institution Press, Washington; London.

WOODMAN, N., TIMM, R.M., SLADE, N.A. \& DOONAN, T.J. 1996. Comparison of traps and baits for censusing small mammals in neotropical lowlands. J. Mammal. 77:274-281. doi:10.2307/1382728

ZORTÉA, M. \& AGUIAR, L. 2008. A diversidade de morcegos conhecida para o Cerrado. In II Simpósio Internacional de Savanas Tropicais. ParlaMundi, Brasília. 


\section{Apêndice 1}

Apêndice 1. Lista dos espécimes coletados nas três localidades amostradas no interior da Estação Ecológica Serra Geral do Tocantins (EESGT). Appendix 1. List of the specimens collected in the three localities surveyed in the Estação Ecológica Serra Geral do Tocantins (EESGT).

\section{Ordem Didelphimorphia}

Família Didelphidae

Caluromys lanatus: APC 1602

Cryptonanus agricolai: APC 1351

Didelphis albiventris: APC 1219, 1352, 1601

Gracilinanus agilis: APC 1376, 1523, 1562

Micoureus demerarae: APC 1241, 1372, 1519

Monodelphis domestica: APC 1350, 1365, 1374, 1585

Thylamys karimii: APC 1197, 1212, 1213, 1229, 1232, 1239, 1248, 1263, 1364, 1514, 1518, 1522, 1559, 1561, 1584, 1588, 1592, 1596, $1618,1620,1668$

Ordem Cingulata

Família Dasypodidae

Cabassous unicinctus: APC 1203, 1632

Ordem Rodentia

Família Cricetidae

Calomys tener: APC 1342, 1370, 1371, 1591, 1615, 1701

Cerradomys marinhus: APC 1373, 1608, 1613, 1671

Cerradomys scotti: APC 1259, 1496

Hylaeamys megacephalus: APC 1366, 1515, 1516, 1521

Necromys lasiurus: APC 1198, 1246, 1254, 1267, 1269, 1494, 1527, 1555, 1556, 1557, 1565, 1589, 1594, 1595, 1605, 1606, 1610, 1616, $1628,1630,1633,1634,1636,1637,1700$

Nectomys rattus: APC 1349, 1379

Oecomys bicolor: APC 1378, 1383, 1532, 1533

Oecomys paricola: APC 1240, 1251, 1260, 1353, 1384

Oligoryzomys fornesi: APC 1196, 1224, 1255

Oligoryzomys moojeni: APC 1216

Oxymycterus delator: APC 1194, 1202, 1211, 1268, 1386, 1517, 1528

Pseudoryzomys simplex: APC 1233

Rhipidomys macrurus: APC 1193, 1381

Thalpomys cerradensis: APC 1199, 1200, 1201, 1207, 1208, 1210, 1214, 1215, 1225, 1226, 1227, 1228, 1230, 1231, 1234, 1243, 1247, $1253,1256,1257,1258,1261,1262,1348,1354,1355,1361,1362,1363,1369,1385,1520,1525,1558,1560,1563,1564,1629,1667$

Família Caviidae

Cavia aperea: APC 1599

Família Echimyidae

Proechimys roberti: APC 1217, 1218, 1235, 1236, 1237, 1242, 1252, 1270, 1382

Thrichomys sp.: APC 1195, 1204, 1205, 1206, 1209, 1220, 1221, 1222, 1223, 1238, 1244, 1245, 1249, 1250, 1264, 1265, 1266, 1343, $1344,145,1346,1347,1359,1360,1367,1368,1375,1377,1380,1493,1495,1497,1499,1524,1526,1583,1586,1587,1590,1593$, 1597, 1598, 1600, 1607, 1609, 1611, 1614, 1617, 1619, 1626, 1627, 1631, 1635, 1669, 1670

Ordem Primates

Família Atelidae Alouatta caraya: APC 1357, 1358

Ordem Artiodactyla

Família Tayassuidae

Tayassu pecari: APC 1192 